\title{
Electrical confinement in a spectrum of two-dimensional Dirac materials with classically integrable, mixed, and chaotic dynamics
}

\author{
Chen-Di Han, ${ }^{1}$ Hong-Ya Xu ${ }^{1}$ and Ying-Cheng Lai ${ }^{1,2, *}$ \\ ${ }^{1}$ School of Electrical, Computer, and Energy Engineering, Arizona State University, Tempe, Arizona 85287, USA \\ ${ }^{2}$ Department of Physics, Arizona State University, Tempe, Arizona 85287, USA
}

(Received 28 October 2019; revised manuscript received 17 December 2019; accepted 20 December 2019; published 3 February 2020)

\begin{abstract}
An emergent class of two-dimensional Dirac materials is $\alpha-T_{3}$ lattices that can be realized by adding an atom at the center of each unit cell of a lattice with $T_{3}$ symmetry. The interaction strength $\alpha$ between this atom and any of its nearest neighbors is a parameter that can be continuously tuned between zero and one to generate a spectrum of materials. We investigate the fundamentally important and practically relevant issue of quasiparticle confinement for the entire spectrum of $\alpha-T_{3}$ materials. Except for the two end points, i.e., $\alpha=0,1$, which correspond to the graphene and pseudospin- 1 lattices, respectively, the time-reversal symmetry is broken, leading to the removal of level degeneracy and facilitating confinement. Taking the approach of quantum scattering off an electrically generated potential cavity in the quantum-dot regime, we characterize confinement by identifying and examining the scattering resonances. We study a number of cavities with characteristically distinct classical dynamics: circular, annular, elliptical, and stadium cavities. For the circular and annular cavities with classically integrable and mixed dynamics, respectively, the scattering matrix can be analytically obtained, so the scattering cross sections and the Wigner-Smith time delay associated with the resonances can be calculated to quantify confinement. For the elliptical and stadium cavities with mixed and chaotic dynamics in the classical limit, respectively, the scattering-matrix approach is infeasible, so we adopt an efficient numerical method to calculate the scattering wave functions and experimentally accessible measures of confinement such as the magnetic moment. The main finding is that, for all the cases, the regime of small $\alpha$ values offers the best confinement possible among the spectrum of $\alpha-T_{3}$ materials, which is general and holds regardless of the nature of the corresponding classical dynamics.
\end{abstract}

DOI: 10.1103/PhysRevResearch.2.013116

\section{INTRODUCTION}

There has been tremendous development of research on two-dimensional (2D) Dirac materials since the experimental realization of graphene [1-4]. A distinct characteristic of lowenergy excitations in 2D Dirac materials is the pseudospin degree of freedom. A variety of such materials have been studied, suggesting that a continuous spectrum of pseudospin quasiparticles may exist in these materials and may be experimentally realized. At the lower end of the spectrum is graphene, whose energy band structure constitutes a pair of Dirac cones with the corresponding low-energy excitations being pseudospin-1/2 particles, which are described by a twocomponent spinor wave function governed by the standard 2D Dirac equation [3]. At the high end of the spectrum are Dirac materials with a $T_{3}$ symmetry, whose energy band contains a pair of Dirac cones and a flat band through the conic intersecting point [5]. Because of the existence of

\footnotetext{
*ying-cheng.lai@asu.edu

Published by the American Physical Society under the terms of the Creative Commons Attribution 4.0 International license. Further distribution of this work must maintain attribution to the author(s) and the published article's title, journal citation, and DOI.
}

three distinct bands, the low-energy excitations need to be described by a spinor wave function of three components, corresponding effectively to pseudospin-1 quasiparticles that obey the Dirac-Weyl equation. In between the pseudospin-1/2 and pseudospin-1 extremes lies a spectrum of pseudospin quasiparticles that can be generated by the corresponding spectrum of $\alpha-T_{3}$ lattices [6-16].

The relativistic quantum behaviors of $\alpha-T_{3}$ particles are described by the generalized Dirac-Weyl equation with a threecomponent spinor, where the original $2 \times 2$ Pauli matrices (for pseudospin-1/2 particles) are replaced by a set of $3 \times 3$ matrices - the set of generalized Pauli matrices. Figure 1(a) illustrates the structure of an $\alpha-T_{3}$ lattice with a $T_{3}$ symmetry, where the unit cell is essentially that of the graphene honeycomb lattice with an additional atom at the center of the hexagon. There are then three nonequivalent atoms in the $\alpha-T_{3}$ unit cell, where $\alpha$ is a parameter characterizing the coupling strength between the central atom and any of the six atoms at the vertices of the hexagon [6]. Especially, if in the graphene lattice the nearest-neighbor interaction energy is $t$, then the interaction energy between the central atom and one on the hexagon is $\alpha t$.

The possible values of $\alpha$ range from zero to one. For $\alpha=0$, the presence of the central atom has no effect on the hexagonal lattice, so the whole lattice effectively reduces to that of graphene with pseudospin- $1 / 2$ quasiparticles. For $\alpha=1$, the 
(a)

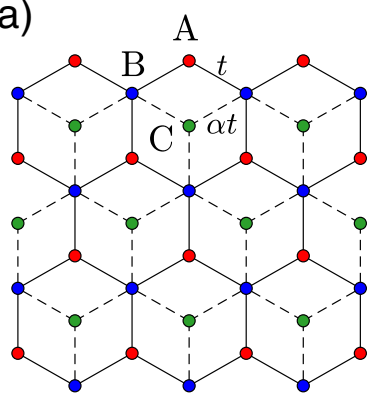

(b)

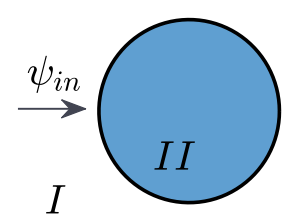

(c)

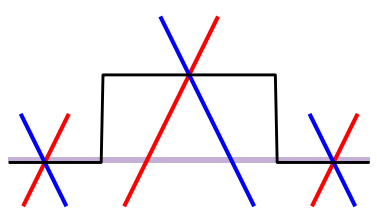

FIG. 1. Illustration of an $\alpha-T_{3}$ lattice, confinement cavity, and the energy band structure. (a) An $\alpha-T_{3}$ lattice, where A, B, and C represent the three nonequivalent atoms, labeled as red, blue, and green circles. The hopping energy between A and B (solid line) is $t$ and between B and C (dashed line) is $\alpha t$. (b) A circular cavity (blue) in an $\alpha-T_{3}$ sheet (gray), which can be realized through an externally applied electric potential. Two geometric regions are specified: one outside and another inside the cavity. (c) For pseudospin-1/2 system $(\alpha=0)$, energy-band structure for scattering, where carriers dominate inside the cavity, forming a quantum-dot structure. The two bands (the pair of vertex-touching Dirac cones) correspond to the two pseudospin states, and the blue and red lines are the linear energy-momentum dispersion relations associated with the two nonequivalent atoms (A and B) in the unit cell, respectively. The horizontal gray line illustrates the incident energy, which is much lower than the potential height.

interaction energy between the central atom and one on the hexagon is identical to that of the nearest neighbor interaction in the graphene lattice, so the $\alpha-T_{3}$ lattice possesses the full $T_{3}$ symmetry with pseudospin- 1 particles. For $\alpha \in(0,1)$, the $\alpha-T_{3}$ lattice is essentially a hybrid between the graphene and pseudospin-1 lattices [8]. For convenience, we call $\alpha$ the pseudospin parameter.

Experimentally, one way to realize $\alpha-T_{3}$ hybrid and pseudospin-1 systems is through photonic crystals [17-19]. The three nonequivalent atoms can be simulated by using coupled waveguides generated by laser inscription [19]. Different lattice constants can be used to build a quantum dot structure [20]. Electronic materials can also be exploited to generate pseudospin-1 lattice systems such as transition-metal oxide $\mathrm{SrTiO}_{3} / \mathrm{SrIrO}_{3} / \mathrm{SrTiO}_{3}$ trilayer heterostructures [21], $\mathrm{SrCu}_{2}\left(\mathrm{BO}_{3}\right)_{2}$ [22], or graphene-in $\mathrm{Te}_{2}$ [23].

In potential device applications of $\alpha-T_{3}$ materials, a fundamental issue is how to effectively confine the quasiparticles in an enclosure. Similar to optics [24], such a structure can be exploited for storage and energy transfer in spintronics and valleytronics $[25,26]$. However, even in a perfectly circular cavity generated by, e.g., an electrostatic potential, confinement of pseudospin- $1 / 2$ particles in graphene is already a nontrivial issue [27], due to the phenomenon of Klein tunneling [28-34]. To confine pseudospin-1 particles is also difficult, due to super-Klein tunneling [33], in which particles can penetrate through a high and wide potential barrier at any angle. Confinement becomes even more difficult in realistic situations where geometric deformations from the circular shape are inevitable, which can lead to chaotic dynamics in the classical limit [35-37]. What are the general features of confining pseudospin quasiparticles of the $\alpha-T_{3}$ lattice in comparison with the confinement of pseudospin- $1 / 2$ and pseudospin-1 particles? The purpose of this paper is to address this question that not only is useful for gaining understanding into the fundamental physics of the quasiparticles in the exotic 2D materials but also has implications to developing future $\alpha-T_{3}$ material based electronic/spintronic devices.

To be concrete, we study confinement of quasiparticles in a cavity in an $\alpha-T_{3}$ lattice, which can be created by applying a step electrostatic potential, the boundary of which divides the lattice system into two regions, as shown in Fig. 1(b). Experimentally, such a structure can be generated by a STM (scanning tunneling microscope) tip induced potential [34,3840] or through the method of doping [41]. The geometric shape of the cavity can be chosen to generate integrable (e.g., a circle), mixed (e.g., an ellipse), or chaotic (e.g., a stadium) dynamics in the classical limit. In order to confine an electron inside the cavity, its energy should be far away from the Klein tunneling regime that occurs for $E \approx U / 2$ for graphene [28-34] and pseudospin-1 materials [33]. For the confinement problem to have physical and applied significance, we focus on the quantum-dot regime where the effect of Klein tunneling is weak $[15,27,42]$. First, we choose the incident energy $E$ such that it is much smaller than the electric potential: $|E| \ll$ $|U|$, as shown by the energy band structure in Fig. 1(c), so as to avoid the Klein tunneling regime. The wave vector inside the cavity is thus much larger than that outside. Second, we choose the size of the cavity such that the system in the quantum-dot regime defined by $k_{0} R \ll 1 \ll V R$, where $k_{0}$ is the wave vector outside, $R$ is the effective size (radius) of the cavity, and $V=U / v_{g}$ is some normalized potential strength (with $v_{g}$ being the group velocity). In this regime, outside of the cavity the wave characteristics of the quasiparticles dominate but inside the cavity the particle nature becomes important - there are then carriers in the cavity. The linear dispersion relation associated with the Dirac cones is $E=v_{g} k_{0}$. The electron motion inside the cavity can be studied in terms of Dirac electron optics [29,34,38,43-67]. As the value of the pseudospin parameter $\alpha$ increases from zero to one, the nature of the quasiparticles of the system changes from pseudospin$1 / 2$ to hybrid and finally to pseudospin- 1 . For $\alpha \neq 0,1$, the time-reversal symmetry ( $T$-symmetry) is broken [11].

The main finding of this paper is that, in the quantumdot regime, among the possible $\alpha-T_{3}$ materials, the strongest or optimal confinement occurs for hybrid materials in between the pseudospin- $1 / 2$ and pseudospin- 1 limits but near the graphene end, i.e., for some value of $\alpha \gtrsim 0$. This result holds not only for the perfectly circular cavity with classical integrable dynamics but also for deformed cavities with mixed or chaotic dynamics in the classical limit. Contributing factors to this phenomenon include $T$-symmetry breaking in the hybrid material and unconventional wave-function behaviors as induced by the boundary conditions.

In Sec. II, we analyze the scattering process from circular cavity for $\alpha-T_{3}$ materials by calculating the quasibound states and the far-field behavior. Especially, because of the circular symmetry, in the quantum-dot regime there are quasibound states with nonzero angular momentum whose lifetime is 
infinite, and the confinement efficacy is determined the number of such states. We find that, for the hybrid system, because of the $T$-symmetry breaking, more such quasibound states can emerge as compared with the graphene and pseudospin-1 limits. In Sec. III, we study the annular cavity for which the scattering process can still be understood analytically. Calculation of the Wigner-Smith time delay for different values of $\alpha$ and different potential profiles indicates that, for certain potential profile, maximum confinement occurs for a small but nonzero value of $\alpha$. Finally, in Sec. IV, we study confinement in deformed cavities with classical mixed and chaotic dynamics, which find applications in enhancing directional emission. In this case, all the quasibound states have a finite lifetime. However, practically, confinement is still possible, which can be characterized by physically measurable quantities such as the magnetic moment.

\section{CONFINEMENT IN A CIRCULAR CAVITY}

We consider the tight-binding model for a free $\alpha-T_{3}$ particle and derive the corresponding continuum Hamiltonian. In the tight-binding framework, the Bloch Hamiltonian of the $\alpha-T_{3}$ lattice system is given by

$$
H(\mathbf{k})=\left(\begin{array}{ccc}
0 & f_{\mathbf{k}} & 0 \\
f_{\mathbf{k}}^{*} & 0 & \alpha f_{\mathbf{k}} \\
0 & \alpha f_{\mathbf{k}}^{*} & 0
\end{array}\right),
$$

where

$$
f_{\mathbf{k}}=-t\left[e^{i k_{y} a_{t}}+2 e^{-i k_{y} a_{t} / 2} \cos \left(\sqrt{3} / 2 k_{x} a_{t}\right)\right]
$$

and $a_{t}$ is the lattice constant. Expanding $f_{\mathbf{k}}$ at zero yields $f_{\mathbf{k}} \approx$ $v_{g}\left(v k_{x}-i k_{y}\right)$, where $v_{g}=3 a_{t} t / 2$ and $v= \pm$ is the valley index. Imposing the normalization $t \rightarrow t / \sqrt{1+\alpha^{2}}$, we get the continuum model for low-energy excitations in the $\alpha-T_{3}$ lattice as

$$
H=v_{g} \boldsymbol{S}^{\alpha} \cdot \boldsymbol{p},
$$

where $S^{\alpha}$ denotes the generalized Pauli matrices that depend on the material parameter $\alpha$. The details for solving the corresponding eigenvalue problem are presented in Appendix A.

Quantum scattering from a circular cavity has been studied for graphene $(\alpha=0)[52,54,68,69]$ and pseudospin-1 $(\alpha=1)$ $[33,70]$ systems. There has also been a study of scattering from a centrally symmetric potential in $\alpha-T_{3}$ materials [16]. Because of the circular symmetry in the potential profile, the scattering problem can be solved analytically.

The Hamiltonian for a general $\alpha-T_{3}$ lattice with a circular cavity is

$$
H=v_{g} \mathbf{S}^{\alpha} \cdot \mathbf{p}+v_{g} V \Theta(R-r),
$$

where $V$ is potential height, $\Theta$ is Heaviside step function, and $\mathbf{S}^{\alpha}$ are the generalized Pauli matrices that depend on the parameter $\alpha$ (detailed in Appendix A). For this Hamiltonian, the three-component spinor wave function can be obtained analytically (Appendix B).

Let $q$ be the wave vector inside the cavity. The linear dispersion relation gives $q v_{g}=k_{0} v_{g}-V$. In the quantum-dot regime $k_{0} R \ll 1 \ll V R$, we have $\left|k_{0}\right| \ll|q|$. In the language of Dirac electron optics, waves inside the cavity will have a large relative refractive index, rendering existent a critical angle for total internal reflection $[15,27,42]$, which makes confinement possible.

There are different ways to characterize confinement quantitatively. For example, we can use the total scattering cross section defined as

$$
\sigma\left(\theta^{\prime}\right)=\oint\left|f\left(\theta, \theta^{\prime}\right)\right|^{2} d \theta
$$

where $\theta^{\prime}$ is the incident angle, $\theta$ specifies the direction of measurement, and $f\left(\theta, \theta^{\prime}\right)$ is determined by the far-field behavior of the scattering wave (Appendix A). A large total cross section corresponds to stronger scattering. Alternatively, the wave function inside the cavity can be used to quantify confinement. With the analytically obtained three-component spinor wave function $\Psi=\left(\psi_{A}, \psi_{B}, \psi_{C}\right)^{T}$ inside the cavity, we have the average density of state (DOS) as

$$
\operatorname{DOS}=\int_{\text {cavity }}\left(\left|\psi_{A}\right|^{2}+\left|\psi_{B}\right|^{2}+\left|\psi_{C}\right|^{2}\right) .
$$

We can normalize the DOS to unit area after integration. In experiments, information about the average DOS can be obtained through conductivity measurement [34,38-40]. Another useful quantity to characterize confinement is the Wigner-Smith time delay $[71,72]$ defined through the scattering matrix $S$ :

$$
\tau=-i \hbar \operatorname{Tr}\left(S^{\dagger} \frac{\partial S}{\partial E}\right) .
$$

A large positive value of $\tau$ is indicative of a confinement state [73].

We consider the quantum-dot regime $k_{0} R \ll 1 \ll V R$ where the incident wave vector is infinitesimally small for a fixed potential height but the wave vector is large inside the cavity. (In terms of the small wavelength inside the cavity, this regime can be called the semiclassical regime.) Figure 2(a) shows, in the parameter plane $\left(\alpha, k_{0} R\right)$, the total scattering cross section $\sigma$ with color-coded values. We see that $\sigma$ exhibits peak values, each corresponding to a quasibound state. The dominant peaks arise in the small $k_{0} R$ regime and depend on the value of $\alpha$ as well. The result in Fig. 2(a) suggests that, in the quantum-dot regime, as the value of $\alpha$ is varied, there can be drastic changes in the quantum states from the point of view of confinement. This can be further seen in Fig. 2(b) that shows, for three different values of $\alpha(\alpha=0,0.1,1), \sigma$ versus $k_{0} R$. While there are peaks in $10^{-1}<k_{0} R<10^{1}$ for all three cases, in the small region $10^{-2}<k_{0} R<10^{-1}$, only the $\alpha=0.1$ case has seen a peak. The existence of a strong quasibound state in $10^{-2}<k_{0} R<10^{-1}$ for $\alpha=0.1$ (but not for $\alpha=0$ or $\alpha=1$ ) is further demonstrated by the average DOS and the Wigner-Smith time delay versus $k_{0} R$, as shown in Figs. 2(c) and 2(d), respectively.

A heuristic understanding of the emergence of strong confinement states associated with $\alpha-T_{3}$ scattering in the hybrid system can be gained by examining the far-field behavior such as backscattering. For graphene, due to the $\pi / 2$ Berry phase, in the low-energy regime backscattering is ruled out $[52,74]$. For pseudospin-1 systems, scattering in the far field is isotropic [33]. What is the far-field behavior in the scattering of $\alpha-T_{3}$ particles for $0<\alpha<1$ ? To address this question, 
(a)

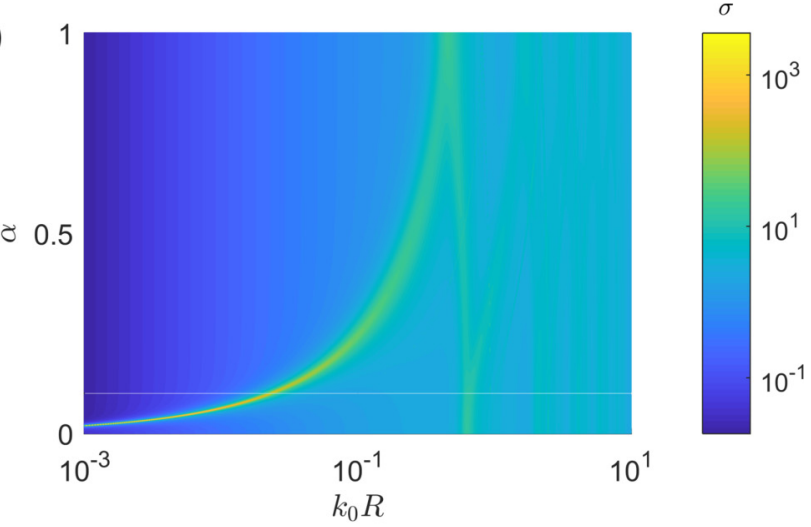

(b)

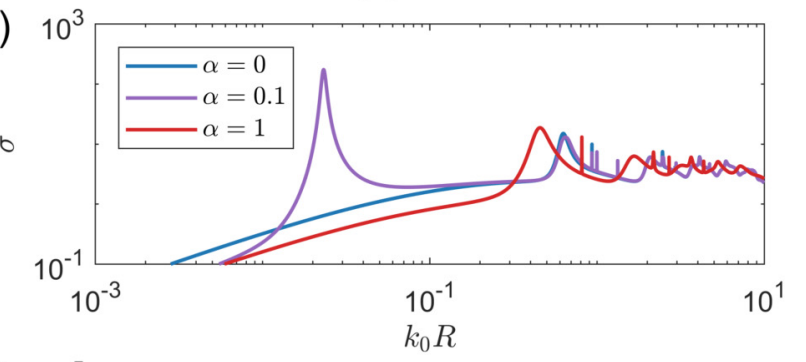

(c)
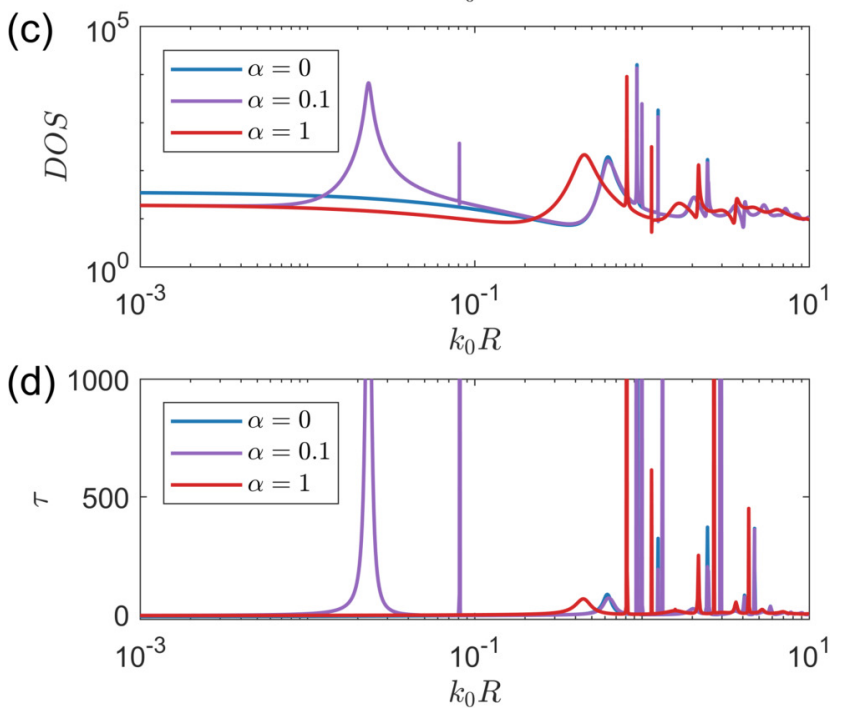

FIG. 2. Scattering of $\alpha-T_{3}$ particles in the regime of small incident wavevectors. The circular electrical cavity has $V R=30$. (a) Color-coded values of the scattering cross section $\sigma$ in the parameter plane $\left(k_{0} R, \alpha\right)$, where a local peak (resonance) in $\sigma$ indicates the existence of a quasibound state and the inverse of the half width of the peak is proportional to the lifetime of the state. There is dominance of resonant peaks in the regime of small $\alpha$ values. [(b)-(d)] Behaviors of $\sigma$, average DOS, and Wigner-Smith time delay versus $k_{0} R$ for three values of $\alpha: 0,0.1$, and 1 . In the region of small $k_{0} R$ values, only the $\alpha=0.1$ case exhibits a strong peak, indicating a superior ability for $\alpha-T_{3}$ material with $\alpha=0.1$ to confine electrons to those of graphene and pseudospin-1 materials.

we analyze different scattering channels. For pseudospin- $1 / 2$ system (graphene), there are two lowest angular momentum states: $\pm 1 / 2$. The counterparts of these states in $\alpha-T_{3}$ scattering in the hybrid system are $l=1$ and $l=0$. Let $A_{l}$ be the scattering wave amplitude with angular momentum $l$. In the semiclassical regime, only the $l=0$ and $l=1$ channels

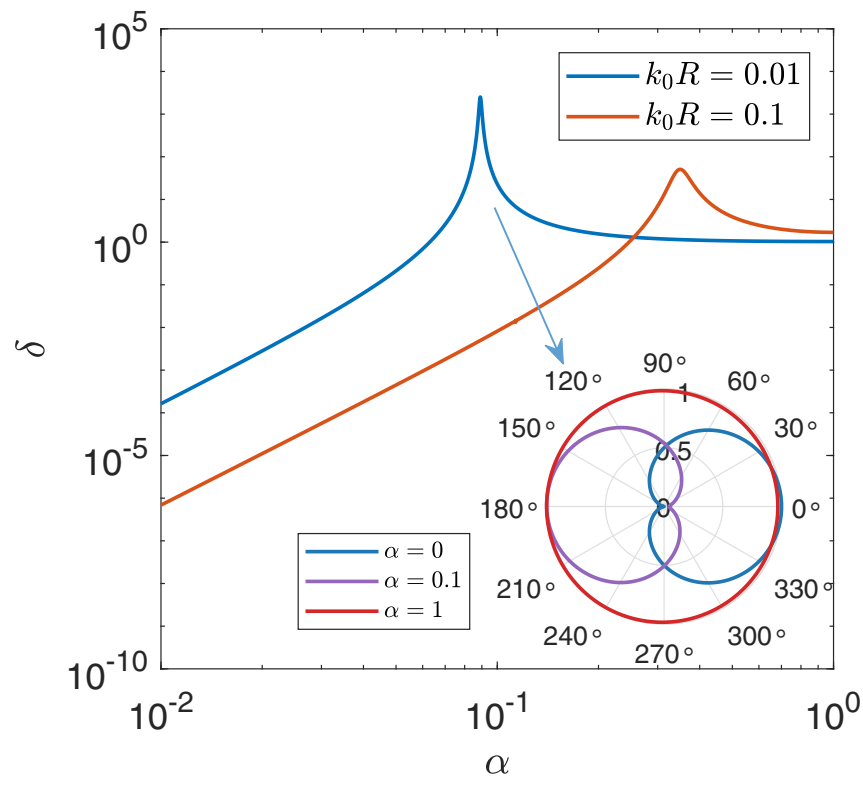

FIG. 3. Enhancement of backscattering from a circular cavity in the hybrid systems. (a) Enhancement ratio $\delta$ vs $\alpha$ for two values of $k_{0} R$. In each case, a peak in $\delta$ arises, signifying strong enhancement of backscattering. For the case of $k_{0} R=0.01$, the peak appears at $\alpha \approx 0.1$ with the enhancement ratio exceeding $10^{3}$. As shown in the inset, a polar representation of the normalized differential cross section for $k_{0} R=0.01$ and $V R=30$, in the low-energy regime, there is no backscattering for pseudospin-1/2 and pseudospin-1 scattering is isotropic. However, in certain hybrid systems, strong backscattering can arise with diminishing forward scattering.

contribute [68] to $A_{l}$. For pseudospin-1 scattering, because of $T$-symmetry preservation, we have $A_{0} \gg A_{1}=A_{-1}$. For the hybrid system $[\alpha \in(0,1)]$, both $A_{0}$ and $A_{1}$ can be appreciable.

In the low-energy regime, the angular scattering cross section is given by

$$
\frac{d \sigma}{d \theta} \approx \frac{2}{\pi k_{0}}\left|A_{0}+A_{1} e^{i \theta}\right|^{2} .
$$

For graphene, the coefficients $A_{0}$ and $A_{1}$ are replaced by $A_{-1 / 2}$ and $A_{1 / 2}$ with $A_{-1 / 2}=A_{1 / 2}$, leading to vanishing scattering cross section for $\theta=\pi$. For pseudospin-1 scattering, the lowest angular momentum mode is $l=0$. In this case, scattering is isotropic [33]. For the hybrid system, in the quantum-dot regime, the dominant scattering channels are still $l=0$ and $l=1$ but, differing from pseudospin- $1 / 2$ or pseudospin- 1 scattering, the scattering channels are dependent upon each other. As a result, backscattering will be enhanced even when the value of $\alpha$ is small (but not equal to zero) (see Appendix B for details).

To characterize enhancement in backscattering, we define the ratio between the values of the angular scattering cross section at angles zero and $\pi$ :

$$
\delta=\frac{d \sigma /\left.d \theta\right|_{\theta=\pi}}{d \sigma /\left.d \theta\right|_{\theta=0}},
$$

as the enhancement factor, which can be calculated numerically from the scattering matrix. Figure 3 shows, for two values of $k_{0} R, \delta$ versus $\alpha$. In each case, there exists a range 


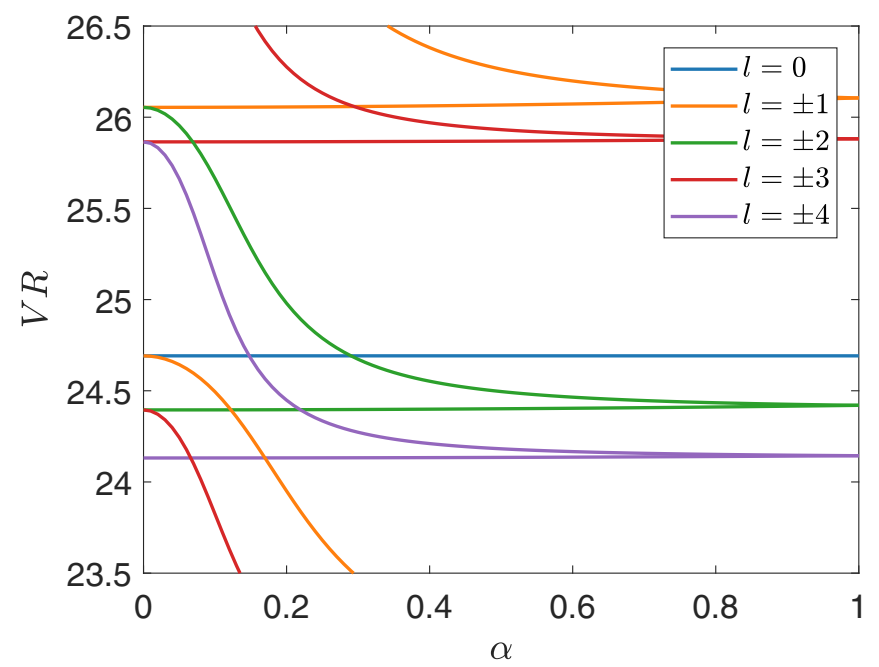

FIG. 4. Quasibound states associated with the first few angular momentum channels in the parameter plane $(\alpha, V R)$. In the limit $\alpha \rightarrow 0$, level degeneracy occurs in the way of $l \rightarrow-l+1$. In the opposite limit $\alpha \rightarrow 1$, degeneracy follows the rule $l \rightarrow-l$. The amount of level separation depends quadratically on $\alpha$ as its value is increased from zero, but the dependence becomes linear as $\alpha$ approaches one. This indicates the emergence of more quasibound states in the small $\alpha$ regime, implying a strong confinement ability there.

of $\alpha$ values in which backscattering is enhanced $(\delta>1)$. Remarkably, in each case there is a peak in $\delta$. For small values of $\alpha$, the enhancement factor is given by

$$
\delta \propto \alpha^{4}\left(k_{0} R\right)^{-2} .
$$

At the peak, we have $\delta \gg 1$, so backscattering is greatly enhanced. This provides an explanation for the emergence of a quasibound state at the corresponding value of $\alpha$.

We examine quasibound states in hybrid material systems in more detail. For plane wave scattering, while there is mixing among scattering associated with different angularmomentum channels as characterized by the scattering matrix, at a resonance scattering depends strongly on the angular momentum $[68,75]$. Let $B_{l}$ be the coefficient of the spinor wave function inside the cavity associated with angular momentum $l$. Each quasibound state corresponds to a maximum in $B_{l}$. For scattering in an infinite plane, the lifetime of the quasibound states can be infinite. The confinement quality is thus determined by the number of quasibound states. To estimate this number for a hybrid system, we begin with the degenerate states for pseudospin-1/2 and pseudospin-1 systems as a result of $T$-symmetry preservation (Appendix B), where the degeneracy is broken for $\alpha \neq 0,1$. The number of quasibound states will then be larger for the hybrid system than for graphene or the pseudospin-1 system.

For $\alpha \rightarrow 0$ and $\alpha \rightarrow 1$, the behavior of broken degeneracy can be studied numerically and analytically. To facilitate numerical computations, we fix the incident angle and vary the potential height $V$. The existence of the confinement states for the first few angular momentum values in the $(\alpha, V R)$ parameter plane are shown in Fig. 4. As the value of $\alpha$ is increased from zero, there is fast separation of the originally degenerate states. For $\alpha \rightarrow 1$, restoration of the degeneracy occurs in a slower manner. Thus, for small values of $\alpha$, we expect a more dramatic deviation in the behaviors of the quantum states from those of graphene.

In the quantum-dot regime, the asymptotic behavior of the scattering wave function associated with each resonance (or quasibound state) can be obtained analytically, e.g., through the method of level doubling [75,76] (Appendix B). The energy separation in the two limiting regimes is given by

$$
\begin{array}{lll}
\Delta_{l} \propto \alpha^{2} l /\left(k_{0} R\right), & \text { for } \alpha \rightarrow 0 & \text { and } l \neq 0, \\
\Delta_{l} \propto(1-\alpha) k_{0} R / l, & \text { for } \alpha \rightarrow 1 & \text { and } l \neq 0 .
\end{array}
$$

For $\alpha \rightarrow 0$, the amount of level separation follows a quadratic dependence on $\alpha$ with the quantity $k_{0} R \ll 1$ in the denominator. As a result, the amount of separation grows quickly as $\alpha$ is increased from zero. Near the pseudospin-1 limit, the amount of the separation has a linear dependence on $k_{0} R(1-\alpha)$ and thus decreases slowly to zero as the value of $\alpha$ approaches one. Numerical support for these analytic estimates is given in Fig. 4.

The quick increase in the number of quasibound states as the value of $\alpha$ is increased from zero implies a strong confinement ability of $\alpha-T_{3}$ materials near the graphene end.

\section{CONFINEMENT IN AN ANNULAR CAVITY}

In the fields of microcavity optics and quantum chaos, annular cavity is a commonly studied type of structures for confinement $[67,77,78]$ because it offers a convenient and systematic way to generate the whole spectrum of classical behaviors ranging from integrable dynamics to chaos. In particular, the system is integrable when the two circles are concentric (ring cavity). Mixed classical dynamics with coexistence of Kolmogorov-Arnold-Moser (KAM) islands and chaos arise when there is a small displacement between the centers of the two circles. Fully developed chaotic dynamics occur when the displacement is sufficiently large. An appealing feature of the annular structure is that the quantum scattering matrix can be analytically calculated for all cases of classical dynamics through a proper coordinate transform [67].

To generate an annular structure on an $\alpha-T_{3}$ sheet, we apply the following electrical potential:

$$
V(\mathbf{r})=V_{1} \Theta\left(R_{1}-r\right) \Theta(|\mathbf{r}-\boldsymbol{\xi}|)+V_{2} \Theta\left(R_{2}-|\mathbf{r}-\boldsymbol{\xi}|\right),
$$

where the two circles have radius $R_{1}$ and $R_{2}$, respectively, and their centers are located at $O$ and $O^{\prime}$ with the displacement vector $\boldsymbol{\xi}=O^{\prime}-O$ between them. A simple change of coordinates gives $\xi=(\xi, 0)$, which aligns the displacement in the $x$ direction. Figures 5(a1)-5(c1) illustrate three representative annular profiles corresponding to three different values of $\xi$. For the integrable dynamics case of $\xi=0$, due to the perfect circular symmetry in the potential, there is no mixing among the states with different angular momenta. (Analytic formulas for the scattering matrix and cross sections for this case are given in Appendix C.) For $\xi \neq 0$, the circular symmetry is broken and the classical dynamics contain a chaotic component. For example, for the potential profile in Fig. 5(b1), we have checked that the classical phase space contains both 

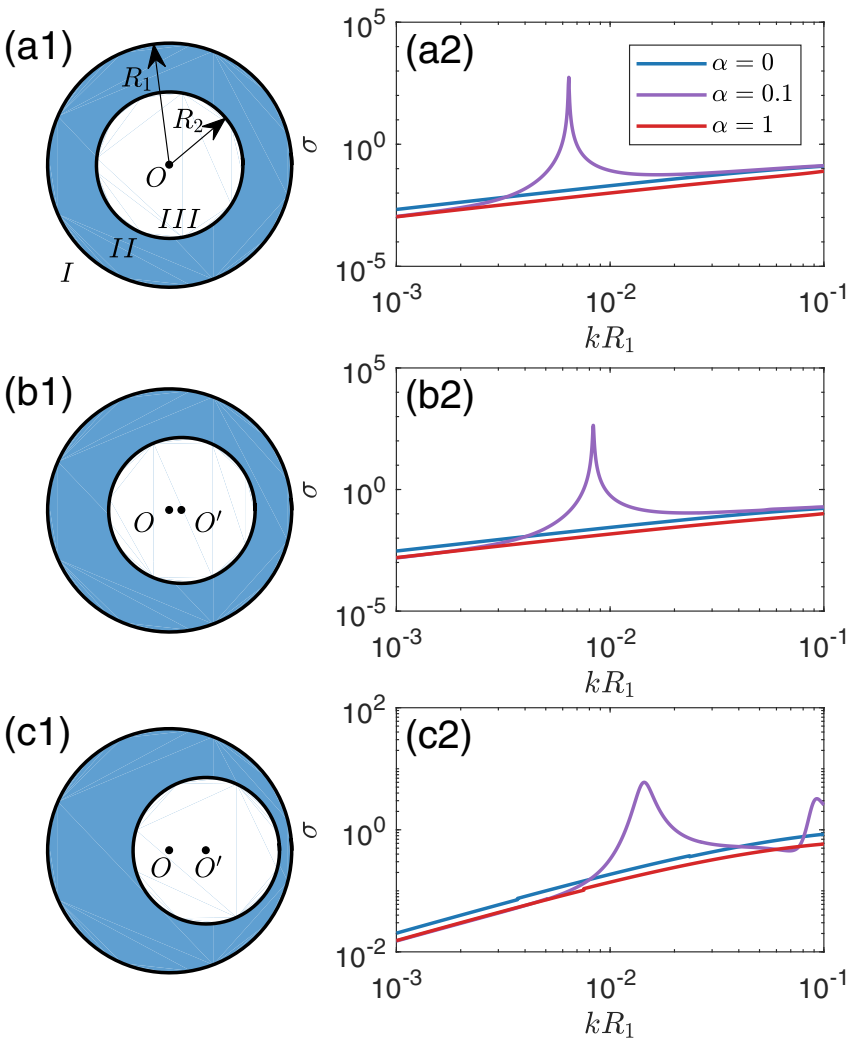

FIG. 5. Three types of annular cavities and the behaviors of the corresponding total scattering cross section. (a1) A ring cavity $(\xi=0)$, where the two boundaries divide the lattice into three regions and the classical dynamics are integrable. (a2) The corresponding total cross section $\sigma$ vs the dimensionless quantity $k_{0} R_{1}$ for different values of $\alpha$ for the parameter setting $R_{2} / R_{1}=0.6$ and $\xi=0$. (b1) Confinement geometry for $\xi R_{1}=0.1$, where $O$ and $O^{\prime}$ are the centers of the outer and inner circles, respectively. In this case, the classical dynamics are mixed. (b2) The corresponding total scattering cross section. (c1) Potential profile for $\xi R_{1}=0.3$, where the classical dynamics are fully chaotic and (c2) the corresponding scattering cross section. For all three types of cavities, there is a resonant peak in the total cross section for $\alpha=0.1$, but no such peak appears for $\alpha=0$ or $\alpha=1$, implying a much stronger ability to confine electrons for the $\alpha-T_{3}(\alpha=0.1)$ cavity than for the graphene or pseudospin-1 cavity. Fully developed classical chaos can smooth out the resonance to some extent, but it is still pronounced.

KAM tori and chaotic regions, but for a larger value of $\xi$ [e.g., the case of Fig. 5(c1)], all KAM tori have been destroyed, leading to fully developed chaos [77]. (Appendix D gives the analytic formulas of some key quantities characterizing relativistic quantum chaotic scattering in this case.)

For convenience, we choose the radius $R_{1}$ of the outer circle as the characteristic length of the system. Figures 5(a2)5(c2) show the total scattering cross section versus the quantity $k_{0} R_{1}$ for the three cases in Figs. 5(a1)-5(c1), respectively, where each panel contains results for three values of $\alpha$ : 0 , 0.1 , and 1 . In all three cases of the potential profile, in the range of $k_{0} R_{1}$ values plotted, for $\alpha=0$ or $\alpha=1$, there is no resonance. However, for $\alpha=0.1$, there is a strong resonant peak about $k_{0} R_{1}=10^{-2}$ for all three cases. The resonance is relatively sharp when the classical dynamics are integrable
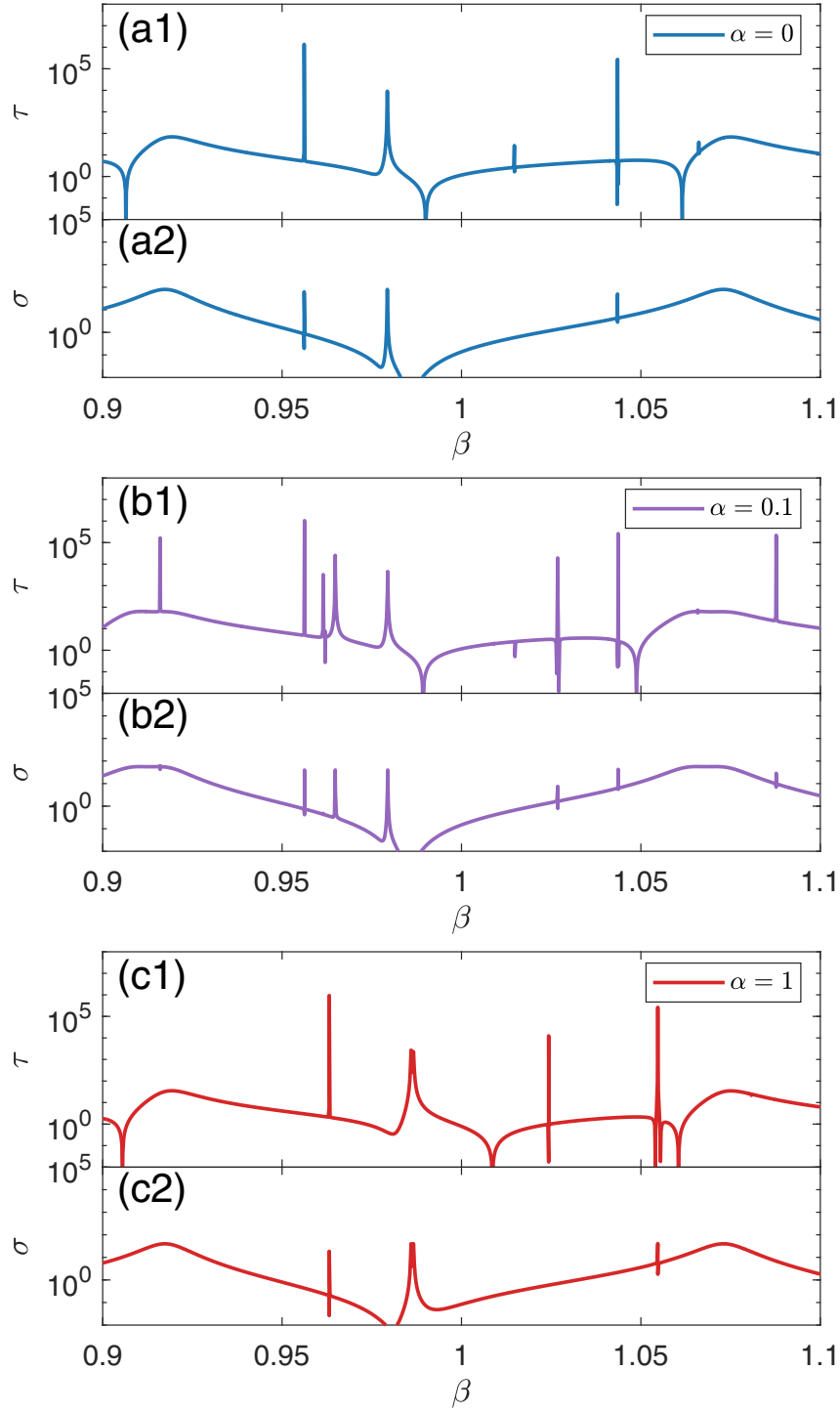

FIG. 6. Comparison of confinement abilities among three material systems in an eccentric annular cavity. Shown are the total cross section and the Wigner-Smith time delay vs the normalized potential height $\beta$ for a fixed incident energy. The parameter setting is $V_{1} R_{1}=-10 \beta$ and $V_{2} R_{1}=40 \beta, k_{0} R_{1}=0.1$, and $\xi R_{1}=0.05$. [(a1)(c1)] Total scattering cross section and [(a2)-(c2)] Wigner-Smith time delay vs $\beta$ for $\alpha=0, \alpha=0.1$, and $\alpha=1$, respectively. Among the three cases, the hybrid system $(\alpha=0.1)$ exhibits more resonant peaks than the other two cases, indicating a stronger confinement ability.

and mixed, as shown in Figs. 5(a2) and 5(b2), respectively. Fully developed chaos can smooth out the resonance to certain extent, but it is still quite pronounced, as shown in Fig. 5(c2).

To characterize the confinement quality for different cavity geometries and for different values of $\alpha$, we fix the incident energy and increase the normalized potential height $\beta$ from zero, which is defined through $V_{1} R_{1}=-10 \beta$ and $V_{2} R_{1}=$ $40 \beta$. Figure 6 shows, for $\xi R_{1}=0.05$ (mixed classical dynamics), the total cross section and the Wigner-Smith time delay versus $\beta$ in the range $\beta \in[0.9,1,1]$ for three values of $\alpha$. In all three cases, there are a number of resonant peaks, each corresponding to a quasibound state, where the inverse 
(a)

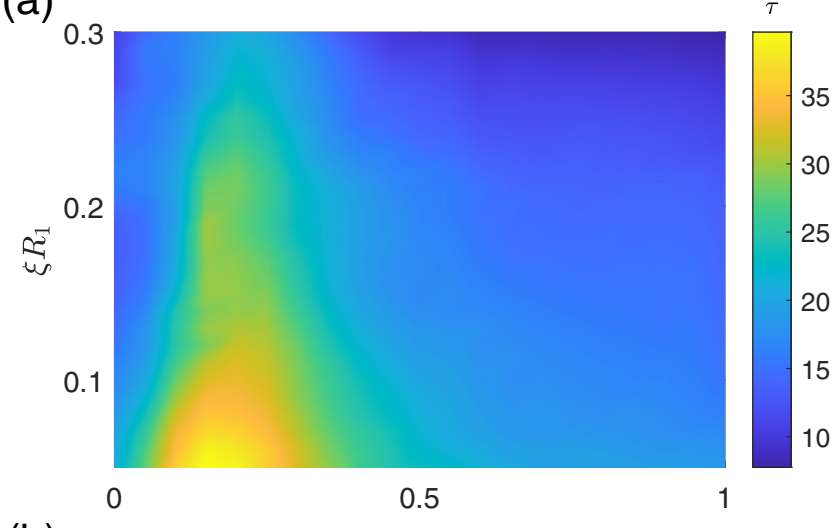

(b)

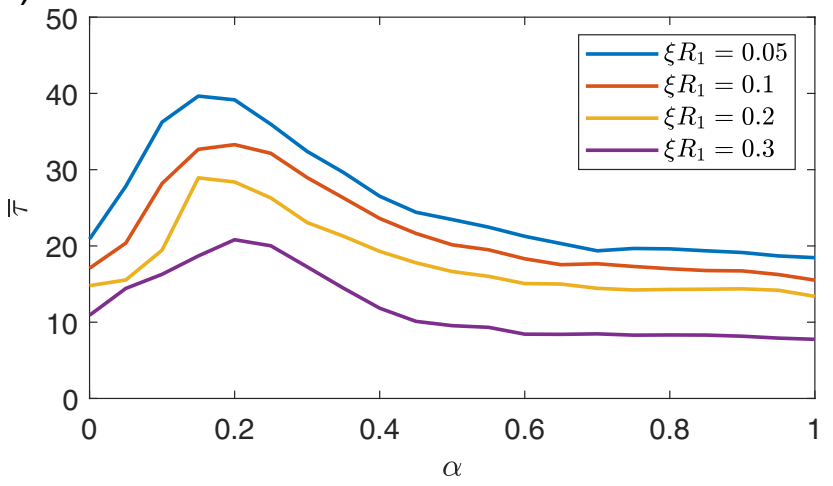

FIG. 7. Confinement for different $\alpha-T_{3}$ materials and different annular cavity geometries. (a) Color-coded value of the integrated Winger-Smith time delay $\bar{\tau}$ in the parameter plane $\left(\alpha, \xi R_{1}\right)$. For $\alpha$ in the vicinity of 0.2 and small values of $\xi, \bar{\tau}$ attains relatively large values. Computationally, the 2D parameter plane is represented by a grid. For each grid point, the integration is carried out with respect to $\beta$ for $\beta \in[0.4,1.6]$. Varying the integration interval does not change the result significantly. (b) The value of $\bar{\tau}$ vs $\alpha$ for four values of the geometric parameter $\xi R_{1}$. Regardless of the geometry, best confinement occurs for $\alpha-T_{3}$ materials with the value of $\alpha$ in the vicinity of 0.2 .

of the width of a resonant peak determines the lifetime of the state. Simply counting the number of resonant peaks in all three cases, we find that the hybrid material system has more as compared with either the graphene or the pseudospin-1 material. The reason, as in the case of a circular cavity, is that, for $\alpha \neq 0,1$, the broken $T$ symmetry makes the originally degenerate states (for $\alpha=0$ or 1 ) nondegenerate.

To further characterize the confinement, we integrate the curves of the Wigner-Smith time delay in Figs. 6(a2)-6(c2)an approach often employed to quantify confinement in optical cavities $[73,79,80]$ :

$$
\bar{\tau}=\frac{1}{\beta_{2}-\beta_{1}} \int_{\beta_{1}}^{\beta_{2}} \tau\left(k_{0}, \beta\right) d \beta .
$$

Roughly, the so-obtained average time delay corresponds to the number of quasibound states contained in the integration interval. To be systematic, we calculate the integral in the parameter plane $\left(\alpha, \xi R_{1}\right)$, as shown in the color-coded graph in Fig. 7(a). We observe a region of relatively higher values of $\bar{\tau}$ (brighter color) for $0<\alpha<0.5$ and $\xi R_{1} \lesssim 0.15$, indicating that a stronger confinement can be achieved for the corresponding lattice structure and cavity geometry. For a fixed geometry, the confinement ability exhibits a nonmonotonous behavior as the value of $\alpha$ is increased from zero to one, as shown in Fig. 7(b) for four different annular cavities.

\section{EFFECT OF GEOMETRIC DEFORMATIONS ON CONFINEMENT}

\section{A. Confinement in deformed cavities with distinct classical dynamics: numerical demonstration}

We have demonstrated that the family of annular cavities, regardless of the corresponding classical dynamics, is able to confine $\alpha-T_{3}$ particles. Can confinement be achieved in more general cavities with their geometrical shape deformed from the circular shape, such as the elliptical cavity with mixed classical dynamics or the stadium-shaped chaotic cavity (a paradigm in the field of quantum chaos [81-83])? (There is quantum chaotic scattering [84-89] in this case.) For pseudospin-1/2 particles, previous studies based on the method of finite-domain scattering (by setting to zero the wave vector outside of the cavity) revealed that confinement modes can exist in the stadium cavity [90-93]. Geometrically, the elliptical and stadium cavities can be generated through continuous deformation of the circular cavity. To quantitatively assess the effect of geometric deformation on confinement, we carry out a comparative analysis of the confinement quality of $\alpha-T_{3}$ particles in the circular, elliptical, and stadium cavities. Here, classical dynamics are defined as those of a free particle moving in a closed billiard system of certain geometry with the spin degree of freedom excluded. Note that, for quantum treatment of $\alpha-T_{3}$ particles based on the Dirac-Weyl equation, the spin degree of freedom is inherently taken into account.

While the scattering of $\alpha-T_{3}$ particles in the circular and annular cavities can be solved analytically, for a deformed cavity without the circular symmetry, analytic solutions are not feasible. In fact, to our knowledge, there were no previous numerical methods for solving the generalized Dirac-Weyl equation for $\alpha-T_{3}$ particles that are neither pseudospin-1/2 nor pseudospin-1. Taking advantage of a recently developed computational method [94] for pseudospin-1 particles based on the multiple multipole (MMP) method in optics [95-99], we have developed an efficient computational method [94] to solve the spinor wave functions associated with the scattering of $\alpha-T_{3}$ particles from an arbitrary geometric domain (Appendix E). The basic idea is to place two sets of fictitious "poles," one inside the cavity and another outside, which are regarded as the sources for generating the scattering wave function. The multiple set of poles (henceforth the term "multiple multipole") can be determined by matching the wave functions at the cavity boundary. From the so-calculated scattering wave functions, we get the density of states (DOS) as a function of some energy-related parameter of the system, where each peak in the DOS corresponds to a quasibound state. (The details of the MMP method for quantum scattering associated with the generalized Dirac-Weyl equation for $\alpha-T_{3}$ particles are given in Appendix E.)

Figure 8 illustrates, for $\alpha=0$ and three types of cavities (circular, elliptical, and stadium-shaped), DOS versus some 

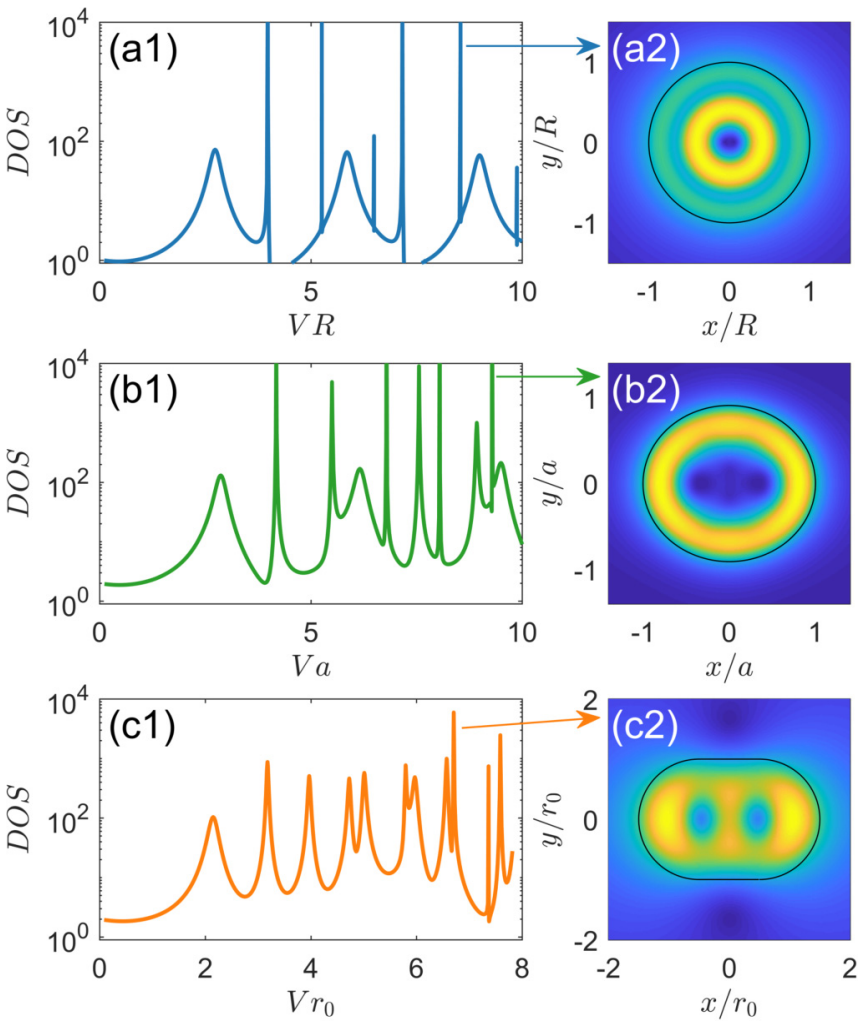

FIG. 8. Resonances and DOS patterns calculated from the MMP method for three types of cavities for pseudospin- $1 / 2$ wave. The top, middle, and bottom rows correspond to the circular, elliptical, and stadium-shaped cavities with classically integrable, mixed, and chaotic dynamics, respectively. For a meaningful comparison, the areas of the cavities are set equal: $\pi a b=\pi R^{2}$ for the ellipse, where $a$ and $b$ are respectively the semimajor and semiminor axes (eccentricity $\gamma=a / b$ ), and $\pi r_{0}^{2}+2 l_{0} r_{0}=\pi R^{2}$ for the stadium, where $l_{0}$ is the length of the straight segment and $r_{0}$ is the radius of the semicircle. In the left column, the normalized energy parameter is $V R$ for the circular cavity, $V a$ for the elliptical cavity, and $V r_{0}$ for the stadium. [(a1)-(c1)] The DOS at an arbitrary point in the cavity vs the energy parameter for the three types of cavities, respectively. In panel (a1), the incident wave vector is $k_{0} R=1 / 10$ and the nearly periodic resonant peaks correspond to different angular momentum states. (a2) A representative DOS pattern associated with a resonant peak, where the square root of the DOS is color-coded. In panel (b1), the eccentricity of the elliptical cavity is $\gamma=1.1$ and the incident wave vector is $k_{0} a=\sqrt{1.1} / 10$. (b2) Color-coded square root of the DOS pattern for a resonant state. In panel $(\mathrm{c} 1)$, the stadium cavity has $A=\pi R^{2}, r_{0} / l_{0}=1$, and $L=2 \pi r_{0}+2 l_{0}$, and the incident wave vector is $k_{0} r_{0}=\sqrt{\pi /(\pi+2)} / 10$. (c2) Color-coded square root of the DOS pattern for a representative resonant state.

normalized energy parameter and the representative DOS patterns in the physical domain corresponding to a pronounced resonant state. The three cavities have the same area. Shown in the left column is the average DOS per unit area inside the cavity versus an energy parameter for the three cavities. In each case, in the interval of the energy parameter, there are a number of distinct peaks in the DOS plot, each corresponding to a quasibound state. The right column shows, for each case, the DOS pattern associated with a typical pronounced resonant peak. Note that, for $\alpha=0$, both components of the spinor wave function are continuous across the cavity boundary. These results, which are obtained from our MMP method, agree with the previous results for scattering of pseudospin-1/2 particles in graphene [90-93].

\section{B. Recurrence of period-2 type of quasibound modes}

In quantum confinement, a fundamental issue is recurrence where, as the wave vector varies, a particular resonant state can arise periodically at a distinct set of energy or wave vector values. In bounded systems, e.g., the stadium cavity with classical chaotic dynamics that has played a paradigmatic role in the study of the phenomenon of scarring in nonrelativistic quantum mechanics [100], scars associated with certain periodic orbits can recur, which can be described by the Gutzwiller formula [81,101]. Recurrence of quantum scars can also arise in graphene billiard systems [102]. In relativistic quantum billiard systems with $T$-symmetry breaking [103], chiral scars of massless spin-1/2 fermions for certain classes of periodic orbits can arise [104-106], which can recur with the energy or the wave vector. In open (scattering) systems with quasibound states, there are still relationships among the classical period orbits, the wave functions, and directional emission in nonrelativistic quantum systems, but the current understanding is that recurrence of the quasibound states is unlikely $[37,107,108]$. Can this conventional wisdom be applied to $\alpha-T_{3}$ particles in a cavity?

Our answer to the above question is surprisingly a "no," as we have found a class of quantum states corresponding to classical periodic orbits of period 2 for $\alpha-T_{3}$ particles which can actually recur. In general, quantum scars are referred to as the unusually high concentrations of the wave function along the classical periodic orbits [100]. In particular, for the elliptical cavity, we find that these modes correspond to the spinor wave functions concentrated along the minor axis of the cavity, as shown in Figs. 9(a) and 9(b) for two cavities with different values of eccentricity, where the insets illustrate the corresponding classical orbits. For convenience, we fix the incident energy $E$ and vary the height $V$ of the electric potential so as to systematically increase the wave vector $q$ inside the cavity. We find that, regardless of the geometric shape of the cavity and of the value of $\alpha$, the period-2 mode can arise repetitively in a periodic fashion. Figure 9(a) shows, for eccentricity value $\gamma=1.1, \alpha=1 / 3$ (red asterisks), and $\alpha=1$ (purple asterisks), the wave-vector value at which the mode emerges versus the index of the recurring mode. A similar plot is displayed in Fig. 9(b) for $\gamma=1.5, \alpha=2 / 3$ (red asterisks), and $\alpha=1$ (purple asterisks). The spinor wavefunction patterns associated with some representative recurring modes are shown on the right-hand side of Figs. 9(a) and 9(b). We find that the spacing in the wave vector for recurrence follows certain rules:

$$
\begin{gathered}
\Delta q=2 \pi / L, \text { for } \alpha=1, \\
\Delta q=\pi / L, \text { for } \alpha \in(0,1),
\end{gathered}
$$

where $L=2 b$ is the length of the minor axis of the elliptical cavity. These resemble the semiclassical rules for quantum scars in closed chaotic systems [81,101]. For $\alpha \neq 1$ in which the $T$ symmetry is broken, the frequency in the wave 

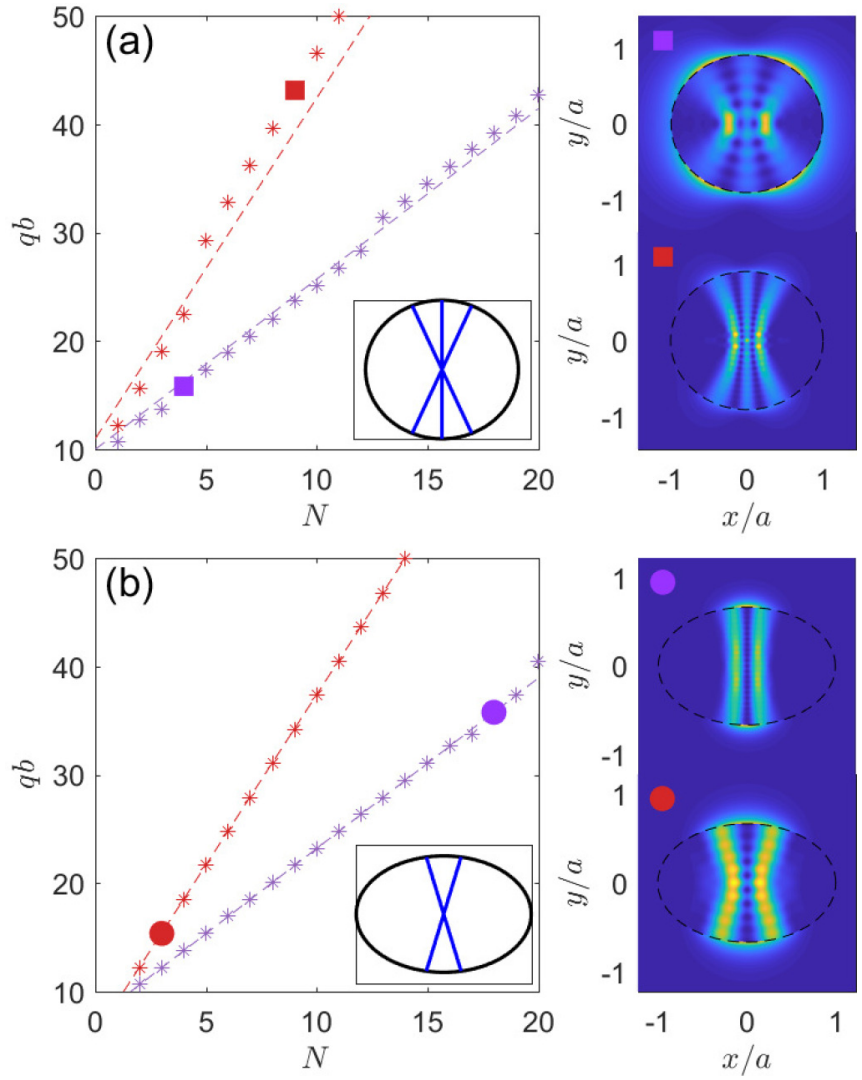

FIG. 9. Recurrence of a class of quasibound state in elliptical cavities. The quasibound state concentrates near the minor axis of the cavity, corresponding to a period- 2 type of classical orbits. (a) Dimensionless wave-vector value $q b$ at which such a quasibound state emerges vs the index of the resonant energy level for $\gamma=1.1$, where two types of $\alpha-T_{3}$ waves are shown: $\alpha=1 / 3$ (purple asterisks) and $\alpha=1$ (red asterisks). Insert: one type of period- 2 orbit with wave function localized along the blue line. (b) Similar plots but for $\gamma=1.5, \alpha=2 / 3$ (purple asterisks) and $\alpha=1$ (red asterisks). The right panels show representative spinor wave function patterns for some specific values of $q$ as indicated in the panels on the left.

vector for the quasibound mode to occur is twice of that for pseudospin-1 system $(\alpha=1)$.

We remark that, in Fig. 8, a few relatively low-excited states are presented. Some relatively high states are shown in Fig. 9. In particular, in Fig. 9(a), we have $q b \approx 40$, where $b$ is the dimension of the cavity and $q$ is the wave vector inside. The setting puts the system in the quantum dot regime, as the wave vector inside the cavity is large. As shown in the right panels of Fig. 9, there are many nodes inside the cavity and the period-2 mode corresponds to a highly exited state. We see that, because of the small wavelength, deformation and classical chaos can have an effect on the states, driving the wave function toward concentrating along some classical periodic orbits.

\section{Characterization of confinement by magnetic moment}

For the circular and annular cavities, we have used the Wigner-Smith delay time and the total scattering cross section to quantify confinement, which can be calculated from the analytic scattering matrix. For a mixed or chaotic cavity, while the scattering wave functions for $\alpha-T_{3}$ particles can be numerically calculated using the MMP method, it does not yield the scattering matrix. For a given state, the degree of confinement is directly related to the peak width of the DOS distribution. However, counting peak numbers and estimating their width are not reliable, especially when the distributions overlap. We thus seek alternative measures to characterize confinement.

One experimentally accessible measure is the magnetic moment [109-111] defined as

$$
\mu_{B}=-\frac{e}{2} \int(\mathbf{r} \times \mathbf{j}) d^{2} \mathbf{r},
$$

where $\mathbf{r}$ is the position, $\mathbf{j}$ is the current, and the integration is with respect to the interior of the cavity. In Ref. [110], it was shown that in topological insulators with impurities, only the states with a large magnetic moment can survive. The robustness of the states against impurities can be measured by the magnetic moment, justifying its use to characterize the degree of confinement.

For the $\alpha-T_{3}$ wave, the associated current is given by $\mathbf{j}=$ $v_{g} \Psi^{\dagger} \mathbf{S}^{\alpha} \Psi$ with the following components in the 2D Cartesian coordinates:

$$
\begin{aligned}
& j_{x}=2 v_{g} \operatorname{Re}\left[\psi_{B}^{*}\left(\psi_{A} \cos \phi+\psi_{C} \sin \phi\right)\right], \\
& j_{y}=-2 v_{g} \operatorname{Im}\left[\psi_{B}^{*}\left(\psi_{A} \cos \phi-\psi_{C} \sin \phi\right)\right] .
\end{aligned}
$$

We compare the confinement properties for three types of cavities with distinct classical dynamics: circular (integrable), elliptical (mixed), and stadium (fully chaotic) cavities. The size parameters for these cavities are the same as those in Fig. 8. We fix the incident wave vector $k_{0} R=0.1$, change the potential height $V$, and calculate the magnetic moment for each quasibound state. Since the current is proportional to the square of the wave function, for each state we normalize the magnetic moment by $|\Psi|^{2}$ to get the average:

$$
\bar{\mu}_{B}=\frac{1}{N} \sum_{i=1}^{N} \frac{\left|\int \mathbf{r} \times \mathbf{j} d^{2} \mathbf{r}\right|}{\int|\Psi|^{2} d^{2} \mathbf{r}},
$$

where the sum is over all quasibound states in a wave-vector range. Figure 10 shows, for the three types of cavities, the average magnetic moment versus $\alpha$. Note that, because of the unbroken $T$ symmetry for $\alpha=0,1$, the net current is zero and so the corresponding average magnetic moment is zero. For $\alpha<0.1$, confinement is strengthened as the value of $\alpha$ is increased. For $\alpha>0.2$, the opposite trend occurs: Confinement is weakened as $\alpha$ becomes larger. For all three cavities, the maximum value of $\bar{\mu}_{B}$ is achieved for $\alpha \approx 0.1$, indicating the strongest possible confinement there among the possible $\alpha-T_{3}$ materials.

Certain features of Fig. 10 can be heuristically understood, as follows. In general, wave functions concentrating along the boundary will contribute to a large magnetic moment and hence strong confinement. For small values of $\alpha$, the third spinor component $\psi_{C}$ is proportional to $\alpha h$ with $h$ being a kind of Bessel function. However, the boundary condition stipulates $h \propto \alpha^{-2}$. As a result, the third component along the boundary is proportional to $\alpha^{-1}$, leading to a larger magnetic moment for small but nonzero values of $\alpha$. Note that, in the 


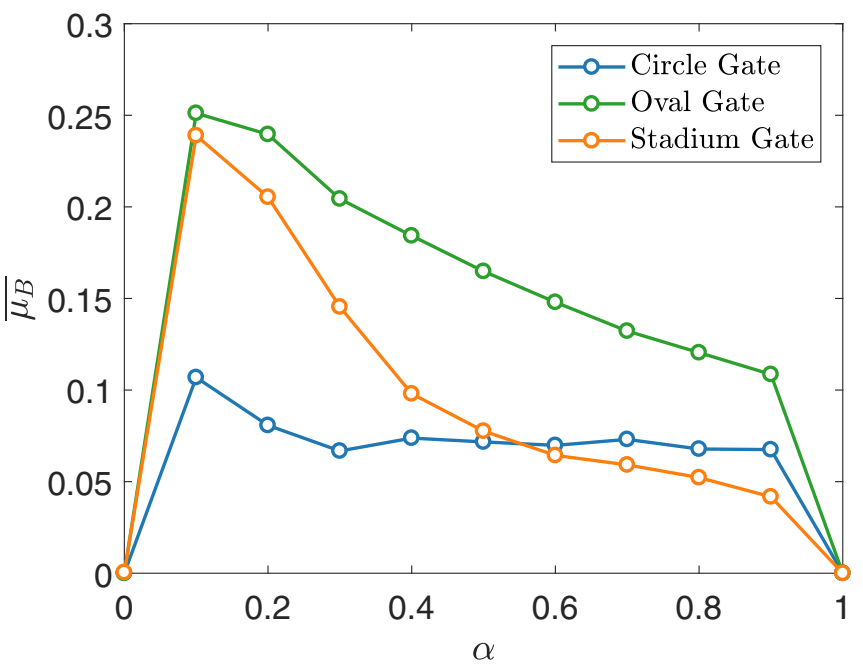

FIG. 10. Average magnetic moments $\bar{\mu}_{B}$ vs $\alpha$ for three different cavities with characteristically distinct classical dynamics. The incident wave vector is set to be $k_{0} R=0.1$ for the circular cavity. The areas of the other two cavities are the same as that of the circular cavity, and the corresponding wave vectors are set as in Fig. 8. The elliptical cavity has eccentricity $\gamma=1.1$ and the parameters of the stadium cavity are $r_{0} / l_{0}=1$ and $L=2 \pi r_{0}+2 l_{0}$. The normalized magnetic moment is averaged for $V R \in[20,40]$, in which there are hundreds of quasibound states for all three cavities. The average magnetic moment is zero for $\alpha=0$ or $\alpha=1$ because of $T$-symmetry preservation, and is maximized for a small but nonzero value of $\alpha$. This indicates that, among the possible $\alpha-T_{3}$ materials, one with the strongest possible confinement occurs in the regime of small (but nonzero) $\alpha$ values.

original definition, Eq. (15), the magnetic moment depends on both the magnitude of the current $\mathbf{j}$ and the position $\mathbf{r}$. While the $T$ symmetry is broken for all three cavities for $\alpha \gtrsim 0$, for the elliptical and stadium cavities, there is one additional broken symmetry: the circular symmetry. As a result, the average magnetic moments are larger than that for the circular cavity.

\section{CONCLUSION AND DISCUSSION}

Given a specific type of quantum materials, the issue of confinement of quasiparticles is of both fundamental importance and applied value. We have addressed the confinement issue in $\alpha-T_{3}$ materials that represent a broad spectrum of state-of-the-art 2D Dirac materials. In terms of the lattice interaction parameter $\alpha$ whose value lies in the unit interval, graphene with pseudospin-1/2 quasiparticles is at the lower end of the spectrum $(\alpha=0)$ while at the other end sits the pseudospin-1 material $(\alpha=1)$. Exactly at the two ends of the spectrum, the $T$ symmetry is preserved, but for any material in between, the symmetry is broken. While experimental realizations of $\alpha-T_{3}$ materials have been achieved only at the two ends of the spectrum, advances in nanotechnologies and materials science may make it possible to create the $2 \mathrm{D}$ Dirac-Weyl materials with arbitrary values of $\alpha$ in the near future. A pertinent theoretical question is thus what kind of $\alpha-T_{3}$ materials would have the best confinement property, especially in the quantum-dot regime. In general, associated with $T$ symmetry breaking is broken level degeneracy, facilitating confinement. It is thus intuitively expected that any $\alpha-T_{3}$ material for $\alpha \neq 0$ would have better confinement properties than graphene and the pseudospin-1 lattice. We have indeed observed this feature. In fact, we have found that optimal confinement is achieved for materials near the graphene end, i.e., those with small and nonzero $\alpha$ values.

The general approach we have undertaken in this study is quantum scattering from a cavity generated by a purely static electric potential. Focusing on the quantum-dot regime, we have studied a number of cavities with characteristically distinct classical dynamics: circular, annular, elliptical, and stadium cavities. For the circular cavity with classical integrable dynamics, the scattering matrix can be analytically obtained, making feasible a confinement analysis based on the scattering cross sections and the Wigner-Smith time delay. Typically, a number of resonant peaks arise in the plots of these quantities versus some energy-related parameter and a relatively sharp resonant peak corresponds to strong confinement, as has been verified by an analysis of backscattering and level separation. For the eccentric annular cavity that can generate the full spectrum of classical dynamics ranging from integrable to chaotic, the scattering matrix can still be analytically calculated through some proper coordinate transform. For the elliptical and stadium cavities, where the classical dynamics are mixed for the former and fully chaotic for the latter, the scattering matrix approach is infeasible. We have adopted an efficient numerical method, the MMP method, to deal with these cavities. In particular, the method enables the three-component spinor wave function for an arbitrary value of $\alpha$ to be numerically computed, based on which experimentally accessible measures of confinement such as the magnetic moment can be calculated. Through analyzing the magnetic moment, we find that, for small $\alpha$ values, for the confined modes, the third component of the spinor wave function is typically larger than the other two components, generating wave functions concentrating along the cavity boundary with a large magnetic moment. For all these cases, we have found that in the region of small $\alpha$ values $(\alpha<0.1)$, confinement is enhanced as the value of $\alpha$ is increased. For $\alpha>0.2$, confinement is weakened with a continuous increase in the value of $\alpha$. Thus, for the $\alpha-T_{3}$ lattice system, the best confinement is achieved for a small but nonzero value of $\alpha$. This phenomenon is general and holds regardless of the nature of the classical dynamics.

\section{ACKNOWLEDGMENTS}

We would like to acknowledge support from the Vannevar Bush Faculty Fellowship program sponsored by the Basic Research Office of the Assistant Secretary of Defense for Research and Engineering and funded by the Office of Naval Research through Grant No. N00014-16-1-2828.

\section{APPENDIX A: POTENTIAL SCATTERING OF $\alpha-T_{3}$ PARTICLES: BASICS}

\section{Free space solutions}

In the free space, the Hamiltonian for $\alpha-T_{3}$ wave is

$$
H=v_{g} \boldsymbol{S}^{\alpha} \cdot \boldsymbol{p},
$$


where $\mathbf{S}^{\alpha}=\left(S_{x}^{\alpha}, S_{y}^{\alpha}\right)$ are $3 \times 3$ generalized Pauli matrices. The two matrices, together with the third one $S_{z}^{\alpha}$, obey the Levi-Civita symbol in three dimensions. For massless quasiparticles in $\alpha-T_{3}$ materials, the matrix $S_{z}^{\alpha}$ does not arise in the Hamiltonian. Letting $\phi=\tan ^{-1} \alpha$, we have

$$
\begin{gathered}
S_{x}^{\alpha}=\left(\begin{array}{ccc}
0 & \cos \phi & 0 \\
\cos \phi & 0 & \sin \phi \\
0 & \sin \phi & 0
\end{array}\right), \\
S_{y}^{\alpha}=-i\left(\begin{array}{ccc}
0 & \cos \phi & 0 \\
-\cos \phi & 0 & \sin \phi \\
0 & -\sin \phi & 0
\end{array}\right) .
\end{gathered}
$$

For $\phi=0, S_{x}^{\alpha}$ and $S_{y}^{\alpha}$ reduce to the $2 \times 2$ Pauli matrices for pseudospin-1/2 particles. For $\phi=\pi / 4$, the Hamiltonian reduces to that for pseudospin-1 particles. For $\alpha \gtrsim 0$, we have $\sin \phi \approx \alpha$.

In the polar coordinates, the eigenequation becomes

$$
\begin{aligned}
& \left(\begin{array}{ccc}
0 & \cos \phi \hat{L}_{-} & 0 \\
\cos \phi \hat{L}_{+} & 0 & \sin \phi \hat{L}_{-} \\
0 & \sin \phi \hat{L}_{+} & 0
\end{array}\right)\left(\begin{array}{c}
\cos \phi h_{l-1} e^{-i \theta} \\
i \kappa h_{l} \\
-\sin \phi h_{l+1} e^{i \theta}
\end{array}\right) e^{i l \theta} \\
& =E\left(\begin{array}{c}
\cos \phi h_{l-1} e^{-i \theta} \\
i \kappa h_{l} \\
-\sin \phi h_{l+1} e^{i \theta}
\end{array}\right) e^{i l \theta},
\end{aligned}
$$

where

$$
\hat{L}_{ \pm}=-i e^{ \pm i \theta}\left(\partial_{r} \pm i \frac{\partial_{\theta}}{r}\right),
$$

and $\kappa=\operatorname{sign}(k)$ is the band index. Comparing with the solutions for the pseudospin-1/2 system, we have that $h_{l}$ are the Bessel type of functions. Let $h_{l}^{(0)}=J_{l}$ be the Bessel function, and $h_{l}^{(1)}=H_{l}^{(1)}$ and $h_{l}^{(2)}=H_{l}^{(2)}$ be the first and second kinds of Hankel functions. Furthermore, we let

$$
{ }^{k} \psi_{l}^{(0,1,2)}(\mathbf{r}) \equiv \frac{1}{\sqrt{2 \pi}}\left(\begin{array}{c}
\cos \phi h_{l-1}^{(0,1,2)}(k r) e^{-i \theta} \\
i \kappa h_{l}^{(0,1,2)}(k r) \\
-\sin \phi h_{l+1}^{(0,1,2)}(k r) e^{i \theta}
\end{array}\right) e^{i l \theta},
$$

where $\mathbf{r}=(r, \theta)$

\section{Boundary conditions}

For a finite potential, we write the three-component spinor wave function as

$$
\Psi(r, \theta)=\left(\begin{array}{c}
\psi_{A} \\
\psi_{B} \\
\psi_{C}
\end{array}\right)=\left(\begin{array}{c}
\mathcal{R}_{A}(r) e^{-i \theta} \\
\mathcal{R}_{B}(r) \\
\mathcal{R}_{C}(r) e^{i \theta}
\end{array}\right) e^{i l \theta},
$$

where $\mathcal{R}_{A}(r), \mathcal{R}_{B}(r)$, and $\mathcal{R}_{C}(r)$ are the corresponding radial components. The eigenequation $H \Psi=E \Psi$ thus becomes

$$
-i v_{g}\left(\begin{array}{ccc}
0 & \cos \phi\left(\frac{d}{d r}+\frac{l}{r}\right) & 0 \\
\cos \phi\left(\frac{d}{d r}-\frac{l-1}{r}\right) & 0 & \sin \phi\left(\frac{d}{d r}+\frac{l+1}{r}\right) \\
0 & \sin \phi\left(\frac{d}{d r}-\frac{l}{r}\right) & 0
\end{array}\right)\left(\begin{array}{l}
\mathcal{R}_{A}(r) \\
\mathcal{R}_{B}(r) \\
\mathcal{R}_{C}(r)
\end{array}\right)=[E-V(r)]\left(\begin{array}{l}
\mathcal{R}_{A}(r) \\
\mathcal{R}_{B}(r) \\
\mathcal{R}_{C}(r)
\end{array}\right) .
$$

Suppose the change in the potential at the cavity boundary $r=R$ is finite. Integrating Eq. (A8) in the infinitesimal interval $r \in[R-\eta, R+\eta]$, where $\eta \sim 0$, we obtain

$$
\begin{aligned}
\mathcal{R}_{B}(R-\eta) & =\mathcal{R}_{B}(R+\eta), \\
\cos \phi \mathcal{R}_{A}(R-\eta)+\sin \phi \mathcal{R}_{C}(R-\eta) & =\sin \phi \mathcal{R}_{A}(R+\eta)+\cos \phi \mathcal{R}_{C}(R+\eta) .
\end{aligned}
$$

Together with the angular part of the spinor wave function, we get the boundary conditions as

$$
\begin{aligned}
\psi_{B}^{<} & =\psi_{B}^{>}, \\
\cos \phi \psi_{A}^{<} e^{i \theta}+\sin \phi \psi_{C}^{<} e^{-i \theta} & =\cos \phi \psi_{A}^{>} e^{i \theta}+\sin \phi \psi_{C}^{>} e^{-i \theta} .
\end{aligned}
$$

where $<$ and $>$ denote the wave functions inside and outside of the boundary, respectively. Note that, across the boundary, the second spinor component must be continuous, but only a linear combination of the first and third components is required to be continuous.

The current density for $\alpha-T_{3}$ particles is

$$
\boldsymbol{j}=v_{g} \Psi^{\dagger} \boldsymbol{S}^{\alpha} \Psi,
$$

whose components are given by

$$
\begin{aligned}
& j_{x}=2 v_{g} \operatorname{Re}\left[\psi_{B}^{*}\left(\psi_{A} \cos \phi+\psi_{C} \sin \phi\right)\right], \\
& j_{y}=-2 v_{g} \operatorname{Im}\left[\psi_{B}^{*}\left(\psi_{A} \cos \phi-\psi_{C} \sin \phi\right)\right] .
\end{aligned}
$$

\section{Elastic scattering theory}

For scattering of $\alpha-T_{3}$ particles from a potential, the general wave function can be written as

$$
\begin{aligned}
\Psi & =\Psi_{\text {in }}+\Psi_{\text {out }} \\
& =\sum_{l} a_{l}\left[{ }^{k} \psi_{l}^{(2)}+\sum_{l^{\prime}} S_{l l^{k}}{ }^{k} \psi_{l^{\prime}}^{(1)}\right] \\
& =\sum_{l} a_{l}\left[2^{k} \psi_{l}^{(0)}+\sum_{l^{\prime}}\left(S_{l l^{\prime}}-\delta_{l l^{\prime}}\right)^{k} \psi_{l^{\prime}}^{(1)}\right],
\end{aligned}
$$

where the incident and reflected waves are expressed as the second and first kinds of Hankel functions, respectively. $S_{l l^{\prime}}$ is the scattering matrix between incident wave with angular momentum $l$ and scattering wave with angular momentum $l^{\prime}$. 
Let $T_{l l^{\prime}} \equiv S_{l l^{\prime}}-\delta_{l l^{\prime}}$ and $\chi_{\text {in }} \equiv \sum_{l} a_{l}{ }^{k} \psi_{l}^{(0)}$. For certain choice of $a_{l}, \chi_{\text {in }}$ corresponds to the plane wave. The far-field behavior of the wave function is

$$
\lim _{k_{0} r \gg 1} \Psi=\chi_{\text {in }}+\frac{f\left(\theta, \theta^{\prime}\right)}{\sqrt{-i r}}\left(\begin{array}{c}
\cos \phi e^{-i \theta} \\
\kappa \\
\sin \phi e^{i \theta}
\end{array}\right) e^{i k r} .
$$

Comparing with the assumption in Eq. (A13), we get

$$
\begin{aligned}
& \frac{f\left(\theta, \theta^{\prime}\right)}{\sqrt{-i r}}\left(\begin{array}{c}
\cos \phi e^{-i \theta} \\
\kappa \\
\sin \phi e^{i \theta}
\end{array}\right) e^{i k r} \\
& \quad=\lim _{k r \gg 1} \sum_{l} a_{l} \sum_{l^{\prime}} T_{l l^{\prime}}\left(\begin{array}{c}
\cos \phi H_{l^{\prime}-1}^{(1)}(k r) e^{-i \theta} \\
i \kappa H_{l^{\prime}}^{(1)}(k r) \\
-\sin \phi H_{l^{\prime}+1}^{(1)}(k r) e^{i \theta}
\end{array}\right) e^{i l^{\prime} \theta} .
\end{aligned}
$$

Using the asymptotic property of the Hankel function [112]

$$
H_{l}^{(1)}(z) \approx \sqrt{\frac{2}{\pi z}} \exp \left[i\left(z-\frac{1}{2} l \pi-\frac{1}{4} \pi\right)\right]
$$

and comparing both sides of Eq. (A15), we get

$$
\begin{aligned}
f_{l}\left(\theta, \theta^{\prime}\right) & =\sqrt{\frac{2}{\pi k}} \sum_{l} \sum_{l^{\prime}} a_{l} T_{l l^{\prime}}(-i)^{l^{\prime}} e^{i l^{\prime} \theta} \\
& =\sqrt{\frac{2}{\pi k}} \sum_{l} f_{l}\left(\theta^{\prime}\right) e^{i l \theta},
\end{aligned}
$$

where

$$
f_{l}\left(\theta^{\prime}\right)=\sum_{m} a_{m} T_{m l}(-i)^{l}
$$

Once the scattering matrix has been obtained, we can analyze the far-field behaviors. For example, the differential cross section is

$$
\frac{d \sigma}{d \theta}=\left|f\left(\theta, \theta^{\prime}\right)\right|^{2}=\frac{2}{\pi k}\left|\sum_{l} f_{l}\left(\theta^{\prime}\right) e^{i l \theta}\right|
$$

and the total scattering cross section is given by

$$
\sigma\left(\theta^{\prime}\right)=\oint d \theta\left|f\left(\theta, \theta^{\prime}\right)\right|^{2}=\frac{4}{k} \sum_{l l^{\prime}} a_{l}\left(T T^{\dagger}\right)_{l l^{\prime}} a_{l^{\prime}}^{*}
$$

\section{APPENDIX B: SCATTERING FROM A CIRCULAR CAVITY}

\section{Solution of scattering wave functions}

The incident wave is

$$
\chi_{\text {in }}(\mathbf{r})=\frac{1}{\sqrt{2}}\left(\begin{array}{c}
\cos \phi \exp \left(-i \theta^{\prime}\right) \\
\kappa_{0} \\
\sin \phi \exp \left(i \theta^{\prime}\right)
\end{array}\right) e^{i k_{0} r \cos \theta},
$$

where $\theta^{\prime}$ is the incident angle and $k_{0}=|E| / v_{g}$ is the incident wave vector. Using the Jacobi-Anger identity [112]

$$
e^{i z \cos \theta} \equiv \sum_{l=-\infty}^{\infty} i^{l} J_{l}(z) e^{i l \theta},
$$

we can expand the plane wave in the polar coordinates as

$$
\begin{aligned}
\chi_{\text {in }}(\mathbf{r}) & =\frac{1}{\sqrt{2}} \sum_{l} i^{l-1}\left(\begin{array}{c}
\cos \phi J_{l-1}\left(k_{0} r\right) e^{i(l-1) \theta} \\
i \kappa_{0} J_{l}\left(k_{0} r\right) e^{i l \theta} \\
-\sin \phi J_{l+1}\left(k_{0} r\right) e^{i(l+1) \theta}
\end{array}\right) \\
& =\sqrt{\pi} \sum_{l=-\infty}^{l=\infty} i^{l-1 k_{0}} \psi_{l}^{(0)} .
\end{aligned}
$$

From Fig. 1, there are two regions. In region I (outside of the cavity), the wave vector is $k$ and the band index is $\kappa_{0}=\operatorname{Sign} E$. Inside the cavity, the wave vector is $q=|E-V| / v_{g}$ and the band index is $\kappa_{1}=\operatorname{Sign}(E-V)$. The wave function in region $\mathrm{I}$ is

$$
\Psi^{(\mathrm{I})}(\mathbf{r})=\chi_{\text {in }}(\mathbf{r})+\sqrt{\pi} \sum_{l=-\infty}^{\infty} i^{l-1} A_{l}^{k_{0}} \psi_{l}^{(1)},
$$

where $A_{l}$ 's are the expansion coefficients. Similarly, in region II, we have

$$
\Psi^{(\mathrm{II})}(\mathbf{r})=\sqrt{\pi} \sum_{l=-\infty}^{\infty} i^{l-1} B_{l}^{q} \psi_{l}^{(0)} .
$$

Imposing boundary conditions and matching the wave functions for each angular momentum channel, we get

$$
\begin{aligned}
B_{l} J_{l}(q R) & =\kappa_{0} \kappa_{1}\left[J_{l}\left(k_{0} R\right)+A_{l} H_{l}^{(1)}\left(k_{0} R\right)\right], \\
B_{l} X_{l}^{(0)}(q R) & =X_{l}^{(0)}\left(k_{0} R\right)+A_{l} X_{l}^{(1)}\left(k_{0} R\right),
\end{aligned}
$$

where

$$
X_{l}^{(0,1,2)}=\cos ^{2} \phi h_{l-1}^{(0,1,2)}-\sin ^{2} \phi h_{l+1}^{(0,1,2)} .
$$

We thus have

$$
\begin{aligned}
& A_{l}=-\frac{J_{l}(q R) X_{l}^{(0)}\left(k_{0} R\right)-\kappa_{0} \kappa_{1} X_{l}^{(0)}(q R) J_{l}\left(k_{0} R\right)}{J_{l}(q R) X_{l}^{(1)}\left(k_{0} R\right)-\kappa_{0} \kappa_{1} X_{l}^{(0)}(q R) H_{l}^{(1)}\left(k_{0} R\right)}, \\
& B_{l}=\frac{H_{l}^{(1)}\left(k_{0} R\right) X_{l}^{(0)}\left(k_{0} R\right)-X_{l}^{(1)}\left(k_{0} R\right) J_{l}\left(k_{0} R\right)}{H_{l}^{(1)}\left(k_{0} R\right) X_{l}^{(0)}(q R)-\kappa_{0} \kappa_{1} X_{l}^{(1)}\left(k_{0} R\right) J_{l}(q R)} .
\end{aligned}
$$

Recalling the definition of the scattering matrix $S^{c d}$

$$
\Psi^{(\mathrm{I})}=\sum_{l=-\infty}^{\infty} a_{l}\left({ }^{k_{0}} \psi_{l}^{(2)}+S_{l}^{c d k_{0}} \psi_{l}^{(1)}\right)
$$

and comparing this with Eq. (B4), we obtain

$$
\begin{aligned}
S_{l}^{c d} & =1+2 A_{l} \\
& =-\frac{J_{l}(q R) X_{l}^{(2)}\left(k_{0} R\right)-\kappa_{0} \kappa_{1} X_{l}^{(0)}(q R) H_{l}^{(2)}\left(k_{0} R\right)}{J_{l}(q R) X_{l}^{(1)}\left(k_{0} R\right)-\kappa_{0} \kappa_{1} X_{l}^{(0)}(q R) H_{l}^{(1)}\left(k_{0} R\right)} .
\end{aligned}
$$

From Eqs. (A19) and (A20), we obtain the differential cross section as

$$
\frac{d \sigma}{d \theta}=\frac{1}{\pi k_{0}}\left|\sum_{l}\left(S_{l}^{c d}-1\right) e^{i l \theta}\right|^{2}
$$

and the following total scattering cross section

$$
\sigma=\frac{2}{k_{0}} \sum_{l}\left|S_{l}^{c d}-1\right|^{2},
$$


where the factor $\left(2 / k_{0}\right)$ is the result of plane wave normalization.

\section{Far-field properties}

In the quantum-dot regime $k_{0} R \ll 1$, for pseudospin- $1 / 2$ scattering the main contribution to the scattering wave function comes from the $\pm 1 / 2$ angular momentum channels. For pseudospin-1 scattering [33], the main contribution comes from the lowest angular momentum channel $l=0$. For $\alpha-T_{3}$ scattering, we have

$$
A_{1}=-\frac{J_{1}(q R) X_{1}^{(0)}\left(k_{0} R\right)-\kappa_{0} \kappa_{1} X_{1}^{(0)}(q R) J_{1}\left(k_{0} R\right)}{J_{1}(q R) X_{1}^{(1)}\left(k_{0} R\right)-\kappa_{0} \kappa_{1} X_{1}^{(0)}(q R) H_{l}^{(1)}\left(k_{0} R\right)} .
$$

Recall the asymptotic properties of the Bessel and Hankel functions [112]:

$$
\begin{gathered}
\lim _{z \rightarrow 0} J_{0}(z) \approx 1, \\
\lim _{z \rightarrow 0} J_{l}(z) \approx \frac{1}{\Gamma(l+1)}\left(\frac{1}{2} z\right)^{l}, \\
\lim _{z \rightarrow 0} H_{0}^{(1)}(z) \approx-H_{0}^{(2)}(z) \approx \frac{2 i}{\pi} \ln (z), \\
\lim _{z \rightarrow 0} H_{l}^{(1)}(z) \approx-H^{(2)}(z) \approx-\frac{i}{\pi} \Gamma(l)\left(\frac{1}{2} z\right)^{-l},
\end{gathered}
$$

for $l>0$. For negative values of $l$, we have $J_{-l}=(-1)^{l} J_{l}$ and $H_{-l}^{(1,2)}=(-1)^{l} H_{l}^{(1,2)}$. We treat $\alpha$ as a perturbation parameter. For $\alpha \gtrsim 0$, the dominant perturbation term is $J_{1}(q R) X_{1}^{(1)}\left(k_{0} R\right)$ in the denominator of Eq. (B13), which has the form $\alpha^{2} H_{2}^{(1)}\left(k_{0} R\right)$. We get

$$
A_{1}=\frac{A_{1}^{(\alpha=0)}}{1+\eta \alpha^{2} /\left(k_{0} R\right)},
$$

where $\eta$ is a parameter. Note that $A_{1}$ decays fast in the quantum-dot regime. The backscattering ratio is given by

$$
\delta=\frac{d \sigma /\left.d \theta\right|_{\theta=\pi}}{d \sigma /\left.d \theta\right|_{\theta=0}}=\frac{\left|A_{0}-A_{1}\right|^{2}}{\left|A_{0}+A_{1}\right|^{2}} \propto \alpha^{4}\left(k_{0} R\right)^{-2} .
$$

\section{Low-field property and level degeneracy}

We analyze the maximum value of $B_{l}$. For $l \neq 0$, the maximum occurs when the denominator of Eq. (B8) vanishes. In the pseudospin- $1 / 2$ case $\alpha=0$, Eq. (B6) becomes

$$
\begin{aligned}
B_{l} J_{l}(q R) & =\kappa_{0} \kappa_{1}\left[J_{l}\left(k_{0} R\right)+A_{l} H_{l}^{(1)}\left(k_{0} R\right)\right], \\
B_{l} J_{l-1}(q R) & =J_{l-1}\left(k_{0} R\right)+A_{l} H_{l-1}^{(1)}\left(k_{0} R\right) .
\end{aligned}
$$

Using the transform $l \rightarrow-l+1$ and noting $J_{-l}=(-1)^{l} J_{l}$ and $H_{-l}^{(1)}=(-1)^{l} H_{l}^{(1)}$, we get $B_{-l+1}=\kappa_{0} \kappa_{1} B_{l}$ and $A_{-l+1}=$ $A_{l}$. For pseudospin-1 ( $\alpha=1$ ) scattering, Eq. (B6) becomes

$$
\begin{aligned}
B_{l} J_{l}(q R)= & \kappa_{0} \kappa_{1}\left[J_{l}\left(k_{0} R\right)+A_{l} H_{l}^{(1)}\left(k_{0} R\right)\right], \\
B_{l}\left(J_{l-1}(q R)-J_{l+1}(q R)\right)= & J_{l-1}\left(k_{0} R\right)-J_{l+1}\left(k_{0} R\right) \\
& +A_{l}\left(H_{l-1}\left(k_{0} R\right)-H_{l+1}\left(k_{0} R\right)\right) .
\end{aligned}
$$

With $l \rightarrow-l$ and using $J_{-l}=(-1)^{l} J_{l}$ and $H_{-l}^{(1)}=(-1)^{l} H_{l}^{(1)}$, we get $A_{-l}=A_{l}$ and $B_{-l}=B_{l}$. The wave function is degenerate with respect to the transform $l \rightarrow-l$.

For a general $\alpha-T_{3}$ system, Eq. (B6) contains the parameter $\alpha$. We have

$$
\begin{aligned}
B_{l} J_{l}(q R) & =\kappa_{0} \kappa_{1}\left[J_{l}\left(k_{0} R\right)+A_{l} H_{l}^{(1)}\left(k_{0} R\right)\right], \\
B_{l} X_{l}^{(0)}(q R) & =X_{l}^{(0)}\left(k_{0} R\right)+A_{l} X_{l}^{(1)}\left(k_{0} R\right) .
\end{aligned}
$$

For $\alpha \gtrsim 0$, we have $\alpha \approx \sin \phi$ and, hence,

$$
X_{l}^{(1)} \approx H_{l-1}^{(1)}\left[1+\eta l(l-1)\left(\frac{\alpha}{k R}\right)^{2}\right]
$$

where $\eta$ is a parameter. In the numerator of $B_{l}, X_{l}^{(1)}\left(k_{0} R\right)$ cancels off $J_{l}\left(k_{0} R\right)$ for small $k_{0} R$ and $l \neq 0$. The denominator has a similar behavior, as in the far-field case:

$$
\begin{aligned}
& H_{l}^{(1)}\left(k_{0} R\right) X_{l}^{(0)}(q R) \\
& \quad=\kappa_{0} \kappa_{1} H_{l-1}^{(1)}\left[1+\eta l(l-1)\left(\frac{\alpha}{k_{0} R}\right)^{2}\right] J_{l}(q R) .
\end{aligned}
$$

To satisfy this equation for small values of $\alpha$, we consider $q \rightarrow q+\Delta_{l}$. Comparing the two sides of the equations, we get

$$
\Delta_{l} \propto \frac{\alpha^{2} l}{k_{0} R}
$$

for $l>0$. For $\alpha$ close to one, we have $\cos \phi \approx \cos (\pi / 4)+$ $(\alpha-1) \sin (\pi / 4)$. Following a similar analysis, we have that $H_{l+1}^{(1)}\left(k_{0} R\right)$ in the denominator is dominant and $H_{l}^{(1)}\left(k_{0} R\right)$ is a perturbation. We get

$$
\Delta_{l} \propto(1-\alpha) \frac{k_{0} R}{l} \quad \text { for }|l|>0
$$

\section{APPENDIX C: SCATTERING OF $\alpha-T_{3}$ WAVE FROM A RING CAVITY}

Consider the ring cavity in Fig. 5(a1), where two boundaries divide the whole space into three regions. The wave vectors in the three regions are $k_{0}=|E| / v_{g}, k_{1}=\left|E-V_{1}\right| / v_{g}$, and $k_{2}=\left|E-V_{2}\right| / v_{g}$ with the respective band indices $\kappa_{0}=$ $\operatorname{sign} E, \kappa_{1}=\operatorname{sign}\left(E-V_{1}\right)$, and $\kappa_{2}=\operatorname{sign}\left(E-V_{2}\right)$. The wave functions in the three regions are given by

$$
\begin{gathered}
\Psi^{(\mathrm{I})}(\mathbf{r})=\sum_{l=-\infty}^{\infty}\left({ }^{k_{0}} \psi_{l}^{(2)}+S_{l}^{\mathrm{ring} k_{0}} \psi_{l}^{(1)}\right), \\
\Psi^{(\mathrm{II})}(\mathbf{r})=\sum_{l=-\infty}^{\infty} C_{l}\left({ }^{k_{1}} \psi_{l}^{(2)}+S_{l}^{c d k_{1}} \psi_{l}^{(1)}\right), \\
\Psi^{(\mathrm{III})}(\mathbf{r})=\sum_{l=-\infty}^{\infty} D_{l}^{k_{2}} \psi_{l}^{(0)} .
\end{gathered}
$$


Let $E_{l}=C_{l} S_{l}^{c d}$. Matching the boundary conditions for each angular momentum channel, we get

$$
\left(\begin{array}{cccc}
\kappa_{1} H_{l}^{(2)}\left(k_{1} R_{2}\right) & -\kappa_{2} J_{l}\left(k_{2} R_{2}\right) & \kappa_{1} H_{l}^{(1)}\left(k_{1} R_{2}\right) & 0 \\
X_{l}^{(2)}\left(k_{1} R_{2}\right) & X_{l}^{(0)}\left(k_{2} R_{2}\right) & X_{l}^{(1)}\left(k_{1} R_{2}\right) & 0 \\
\kappa_{1} H_{l}^{(2)}\left(k_{1} R_{1}\right) & 0 & \kappa_{1} H_{l}^{(1)}\left(k_{1} R_{1}\right) & -\kappa_{0} H_{l}^{(1)}\left(k_{0} R_{1}\right) \\
X_{l}^{(2)}\left(k_{1} R_{1}\right) & 0 & X_{l}^{(1)}\left(k_{1} R_{1}\right) & -X_{l}^{(1)}\left(k_{0} R_{1}\right)
\end{array}\right)\left(\begin{array}{c}
C_{l} \\
D_{l} \\
E_{l} \\
S_{l}^{\text {ring }}
\end{array}\right)=\left(\begin{array}{c}
0 \\
0 \\
\kappa_{0} H_{l}^{(2)}\left(k_{0} R_{1}\right) \\
X_{l}^{(2)}\left(k_{0} R_{1}\right)
\end{array}\right) .
$$

The solutions of the coefficients are

$$
\begin{aligned}
C_{l} & =\kappa_{0} \kappa_{1} \frac{H_{l}^{(2)}\left(k_{0} R_{1}\right)+H_{l}^{(1)}\left(k_{0} R_{1}\right) S_{l}^{c d}}{H_{l}^{(2)}\left(k_{1} R_{1}\right)+H_{l}^{(1)}\left(k_{1} R_{1}\right) S_{l}^{c d}}, \\
D_{l} & =\kappa_{0} \kappa_{1} C_{l} \frac{H_{l}^{(2)}\left(k_{1} R_{2}\right)+H_{l}^{(1)}\left(k_{1} R_{2}\right) S_{l}^{c d}}{J_{l}\left(k_{2} R_{2}\right)} .
\end{aligned}
$$

We thus get the scattering matrix for the ring cavity as

$$
S_{l}^{\mathrm{ring}}=-\frac{\kappa_{0} x_{l} H_{l}^{(2)}\left(k_{0} R_{1}\right)-\kappa_{1} y_{l} X_{l}^{(2)}\left(k_{0} R_{1}\right)}{\kappa_{0} x_{l} H_{l}^{(1)}\left(k_{0} R_{1}\right)-\kappa_{1} y_{l} X_{l}^{(1)}\left(k_{0} R_{1}\right)},
$$

where

$$
\begin{aligned}
& x_{l}=X_{l}^{(2)}\left(k_{1} R_{1}\right)+X_{l}^{(1)}\left(k_{1} R_{1}\right) S_{l}^{c d}, \\
& y_{l}=H_{l}^{(2)}+H_{l}^{(1)}\left(k_{1} R_{1}\right) S_{l}^{c d},
\end{aligned}
$$

with $S_{l}^{c d}$ given by Eq. (B10).

\section{APPENDIX D: SCATTERING OF $\alpha-T_{3}$ WAVE FROM AN ECCENTRIC CIRCULAR CAVITY}

Similar to scattering from a ring cavity, in an eccentric circular cavity there are three distinct regions with wave vectors $k_{0}, k_{1}$, and $k_{2}$. Because of the eccentricity, there is mixing of wave functions from different angular-momentum channels. We can write

$$
\begin{gathered}
\Psi^{(\mathrm{I})}(\mathbf{r})=\sum_{l=-\infty}^{\infty} a_{l}^{0}\left[{ }^{k_{0}} \psi_{l}^{(2)}+\sum_{l^{\prime}=-\infty}^{\infty} S_{l l^{\prime}}{ }^{{ }^{0}} \psi_{l^{\prime}}^{(1)}\right], \\
\Psi^{(\mathrm{II})}(\mathbf{r})=\sum_{l=-\infty}^{\infty} \sum_{m=-\infty}^{\infty}{ }^{l} a_{m}^{1}\left[{ }^{k_{1}} \psi_{l}^{(2)}+\sum_{m^{\prime}=-\infty}^{\infty} S_{m m^{\prime}}^{o d}{ }^{k_{1}} \psi_{l^{\prime}}^{(1)}\right],
\end{gathered}
$$

where $S^{o d}$ is the scattering matrix for the inner circle, a transformed version of the inner circle in the corresponding ring cavity:

$$
S^{o d}=U^{-1} S^{c d} U
$$

where the transformation matrix is $U=\left[U_{l \mu}\right]=\left[J_{\mu-l}\left(k_{1} \xi\right)\right]$ and its inverse is $U^{-1}=\left[U_{m l}^{-1}\right]=\left[J_{m-l}\left(k_{1} \xi\right)\right]$. We match the boundary conditions

$$
\begin{aligned}
& a_{l}^{0} X_{l}^{(2)}\left(k_{0} R_{1}\right) \delta_{l m}+a_{l}^{0} S_{l m} X_{m}^{(1)}\left(k_{0} R_{1}\right) \\
& \quad={ }^{l} a_{m}^{1} X_{m}^{(2)}\left(k_{1} R_{1}\right)+\sum_{j}{ }^{l} a_{j}^{1} S_{j m}^{o d} X_{m}^{(1)}\left(k_{1} R_{1}\right), \\
& i \kappa_{0}\left[a_{l}^{0} H_{l}\left(k_{0} R_{1}\right) \delta_{l m}+a_{l}^{0} S_{l m} H_{m}^{(1)}\left(k_{0} R_{1}\right)\right] \\
& \quad=i \kappa_{1}\left[{ }^{l} a_{m}^{1} H_{m}^{(2)}\left(k_{1} R_{1}\right)+\sum_{j}{ }^{l} a_{j}^{1} S_{j m}^{o d} H_{m}^{(1)}\left(k_{1} R_{1}\right)\right],
\end{aligned}
$$

and define

$$
\begin{aligned}
\mathbb{A}^{0} & =\left[a_{l}^{0} \delta_{l m}\right], \quad \mathbb{A}=\left[{ }^{l} a_{m}^{1}\right], \\
\mathbb{C}^{(1,2)} & =\left[X_{l}^{(1,2)}\left(k_{0} R_{1}\right) \delta_{l m}\right], \quad \mathbb{D}^{(1,2)}=\left[H_{l}^{(1,2)}\left(k_{0} R_{1}\right) \delta_{l m}\right], \\
\mathbf{c}^{(1,2)} & =\left[X_{l}^{(1,2)}\left(k_{1} R_{1}\right) \delta_{l m}\right], \quad \mathbf{d}^{(1,2)}=\left[H_{l}^{(1,2)}\left(k_{1} R_{1}\right) \delta_{l m}\right] .
\end{aligned}
$$

Equation (D4) can be rewritten as a matrix equation

$$
\begin{aligned}
\mathbb{A}^{0} \mathbb{C}^{(2)}+\mathbb{A}^{0} S \mathbb{C}^{(1)} & =\mathbb{A} \mathbf{c}^{(2)}+\mathbb{A} S^{o d} \mathbf{c}^{(1)}, \\
\kappa_{0}\left[\mathbb{A}^{0} \mathbb{D}^{(2)}+\mathbb{A}^{0} S \mathbb{D}^{(1)}\right] & =\kappa_{1}\left[\mathbb{A} \mathbf{d}^{2}+\mathbb{A} S^{o d} \mathbf{d}^{(1)}\right] .
\end{aligned}
$$

We obtain

$$
S=-\frac{\mathbb{D}^{(2)}-\kappa_{0} \kappa_{1} \mathbb{D}^{(2)} \mathbb{E}}{\mathbb{D}^{(1)}-\kappa_{0} \kappa_{1} \mathbb{D}^{(1)} \mathbb{E}},
$$

where $\mathbb{E}=\mathbb{F}^{-1} \mathbb{D}, \mathbb{F}$, and $\mathbb{G}$ are defined as

$$
\begin{aligned}
& \mathbb{F}=\mathbf{c}^{(2)}+S^{o d} \mathbf{c}^{(1)}, \\
& \mathbb{G}=\mathbf{d}^{(2)}+S^{o d} \mathbf{d}^{(1)},
\end{aligned}
$$

and

$$
\mathbb{A}=\frac{\mathbb{A}^{0} \mathbb{C}^{(2)}+\mathbb{A}^{0} S \mathbb{C}^{(1)}}{\mathbf{c}^{(2)}+S^{c d} \mathbf{c}^{(1)}}
$$

with $\mathbb{A}^{0}$ being the coefficient for the incident wave.

\section{APPENDIX E: MMP METHOD FOR SOLVING $\alpha-T_{3}$ WAVE SCATTERING FROM AN ARBITRARY DOMAIN}

We describe a generalized MMP method that can be used to solve the scattering of $\alpha-T_{3}$ wave in an efficient way. The method was originated in optics [95-99] and recently adopted for pseudospin-1 wave scattering [94]. Consider the simple case of a single scattering cavity, where the regions outside of and inside it are denoted as I and II, respectively, with wave vectors $k_{0}$ and $q$ as well as band indices $\kappa_{0}$ and $\kappa_{1}$. Suppose a plane spinor wave is incident on the cavity, which can be written as

$$
\chi^{\text {in }}(\mathbf{r})=\left(\begin{array}{c}
\chi_{A}^{\text {in }} \\
\chi_{B}^{\text {in }} \\
\chi_{C}^{\text {in }}
\end{array}\right)=\frac{1}{\sqrt{2}}\left(\begin{array}{c}
\cos \phi \exp \left(-i \theta^{\prime}\right) \\
\kappa_{0} \\
\sin \phi \exp \left(i \theta^{\prime}\right)
\end{array}\right) e^{i k_{0} r \cos \theta} .
$$

To calculate the scattering wave function, we place a number of "poles" inside and outside of the cavity, as shown in Fig. 11. Poles inside and outside of the cavity are labeled as $m_{\mathrm{II}}$ and $m_{\mathrm{I}}$, respectively, with their total numbers $M_{\mathrm{II}}$ and $M_{\mathrm{I}}$. Each pole is treated as a fictitious source. To avoid the problem of singularity at a source, the scattering wave function outside of the cavity is given as the superposition of the waves from all the poles inside the cavity, while the wave function inside 

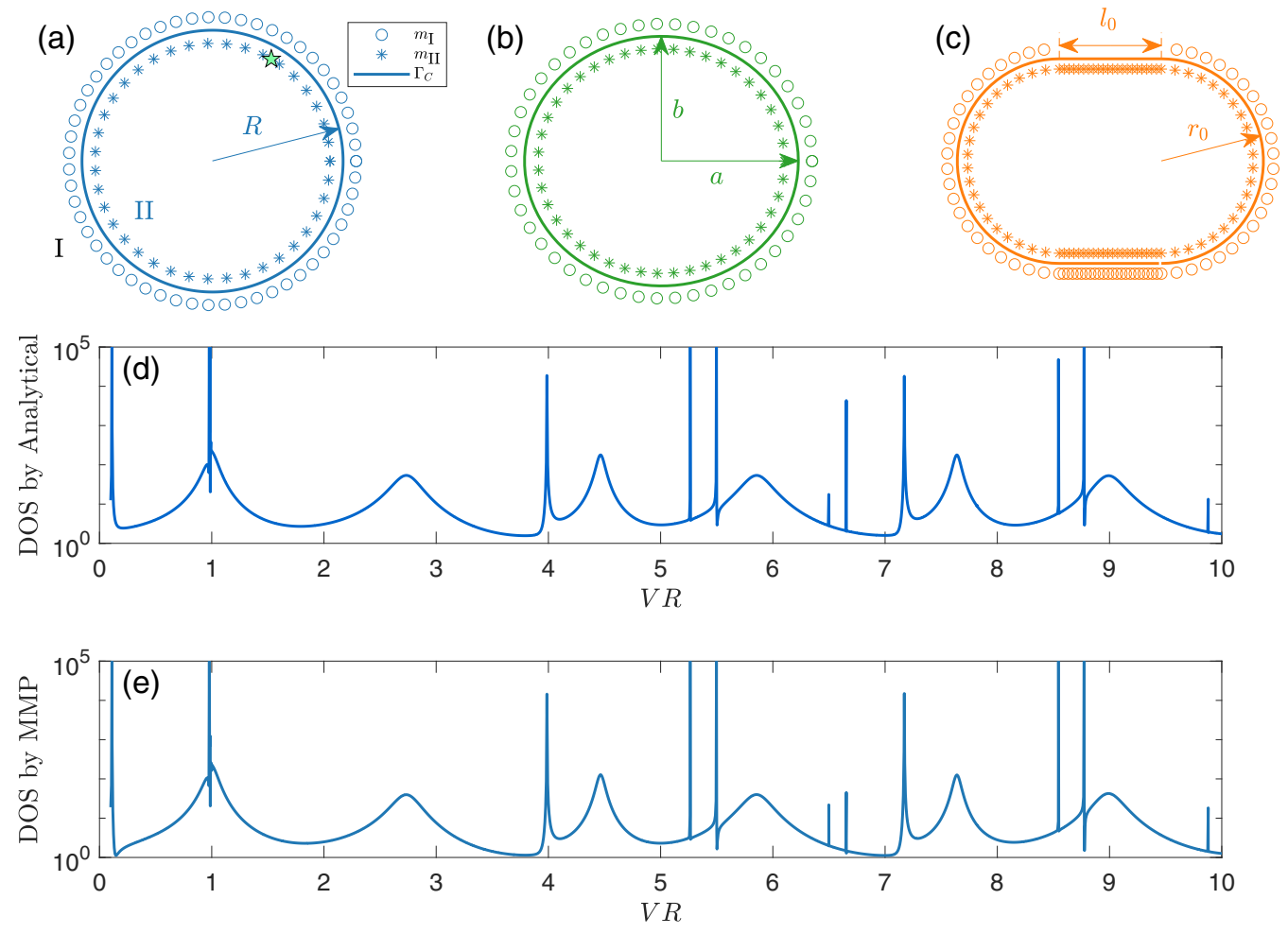

FIG. 11. Illustration of MMP method for $\alpha-T_{3}$ wave scattering and validation. [(a)-(c)] Distributions of interior and exterior poles for the circular, elliptical, and stadium cavities, respectively. For each cavity, the solid curve represents the cavity boundary $\Gamma$, and the regions outside of and inside the cavity are labeled as I and II, respectively. (d) For the circular cavity, analytically obtained local density of state (LDOS) vs the potential parameter $V R$ at the test point $(r=0.9$ and $\theta=\pi / 3)$ specified by the green pentagram in panel (a). (e) The LDOS at the same point obtained from MMP. The agreement between the results in panels (d) and (e) is excellent.

the cavity is determined by the poles outside, as illustrated in Figs. 11(a)-11(c). For a fictitious source, the base function is

$$
\Psi_{l}(\mathbf{r})=\frac{1}{\sqrt{2 \pi}}\left(\begin{array}{c}
\cos \phi h_{l-1}^{(0,1,2)}(k r) e^{-i \theta} \\
i \kappa h_{l}^{(0,1,2)}(k r) \\
-\sin \phi h_{l+1}^{(0,1,2)}(k r) e^{i \theta}
\end{array}\right) e^{i l \theta},
$$

where $h_{l}^{(0,1,2)}(z)$ denotes some type of Bessel function. For each source, we choose the outgoing wave as the basis, i.e., the Hankel function of the first kind: $H_{l}^{(1)}$. The wave function outside of the cavity can be written in terms of the poles inside as

$$
\begin{aligned}
& \Psi^{(\mathrm{I})}(\mathbf{r})=\left(\begin{array}{c}
\psi_{A}^{(\mathrm{I})} \\
\psi_{B}^{(\mathrm{I})} \\
\psi_{C}^{(\mathrm{I})}
\end{array}\right) \\
& =\sum_{m_{\mathrm{II}}} \sum_{l} F_{l}^{m_{\mathrm{II}}}\left(\begin{array}{c}
\cos \phi H_{l-1}^{(1)}\left(k_{0} d_{m_{\mathrm{II}}}\right) e^{-i \theta_{m_{\mathrm{II}}}} \\
i \kappa_{0} H_{l}^{(1)}\left(k_{0} d_{m_{\mathrm{II}}}\right) \\
-\sin \phi H_{l+1}^{(1)}\left(k_{0} d_{m_{\mathrm{II}}}\right) e^{i \theta_{m_{\mathrm{II}}}}
\end{array}\right) e^{i l \theta_{m_{\mathrm{II}}}},
\end{aligned}
$$

where

$$
\begin{aligned}
d_{m_{\mathrm{II}}} & \equiv\left|\mathbf{d}_{m_{\mathrm{II}}}\right|=\left|\mathbf{r}-\mathbf{r}_{m_{\mathrm{II}}}\right|, \\
\theta_{m_{\mathrm{II}}} & \equiv \operatorname{Angle}\left(\mathbf{r}-\mathbf{r}_{m_{\mathrm{II}}}\right),
\end{aligned}
$$

and $F_{l}^{m_{\mathrm{II}}}$ is the expansion coefficient for the eigenvector from the $m_{\mathrm{II}}$ th pole with angular momentum index $l$ located at $\mathbf{r}_{m_{\mathrm{II}}}$.
Similarly, the wave function inside the cavity is determined by the poles outside of the cavity:

$$
\begin{aligned}
\Psi^{(\mathrm{II})}(\mathbf{r}) & =\left(\begin{array}{c}
\psi_{A}^{(\mathrm{II})} \\
\psi_{B}^{(\mathrm{II})} \\
\psi_{C}^{(\mathrm{II})}
\end{array}\right) \\
& =\sum_{m_{\mathrm{I}}} \sum_{l} F_{l}^{m_{\mathrm{I}}}\left(\begin{array}{c}
\cos \phi H_{l-1}^{(1)}\left(q d_{m_{\mathrm{I}}}\right) e^{-i \theta_{m_{\mathrm{I}}}} \\
i \kappa_{1} H_{l}^{(1)}\left(q d_{m_{\mathrm{I}}}\right) \\
-\sin \phi H_{l+1}^{(1)}\left(q d_{m_{\mathrm{I}}}\right) e^{i \theta_{m_{\mathrm{I}}}}
\end{array}\right) e^{i l \theta_{m_{\mathrm{I}}}}
\end{aligned}
$$

where

$$
d_{m_{\mathrm{I}}} \equiv\left|\mathbf{d}_{m_{\mathrm{I}}}\right|=\left|\mathbf{r}-\mathbf{r}_{m_{\mathrm{I}}}\right|, \quad \text { and } \quad \theta_{m_{\mathrm{I}}} \equiv \operatorname{Angle}\left(\mathbf{r}-\mathbf{r}_{m_{\mathrm{I}}}\right) \text {. }
$$

To match the boundary conditions, the three components of the spinor wave function must satisfy

$$
\begin{aligned}
& \left.\left(\chi_{B}^{\text {in }}+\psi_{B}^{(\mathrm{I})}\right)\right|_{\mathbf{r} \in \Gamma}=\left.\psi_{B}^{(\mathrm{II})}\right|_{\mathbf{r} \in \Gamma}, \\
& {\left.\left[\cos \phi\left(\chi_{A}^{\text {in }}+\psi_{A}^{(\mathrm{I})}\right)+\sin \phi\left(\chi_{C}^{\text {in }}+\psi_{C}^{(\mathrm{I})}\right)\right]\right|_{\mathbf{r} \in \Gamma}} \\
& =\left.\left(\cos \phi \psi_{A}^{(\mathrm{II})}+\sin \phi \psi_{C}^{(\mathrm{II})}\right)\right|_{\mathbf{r} \in \Gamma^{\circ}} .
\end{aligned}
$$

Expanding this equation in terms of the poles and the corresponding eigenvectors, we obtain

$$
\begin{aligned}
& \sum_{m_{\mathrm{II}}} \sum_{l}{ }^{j} P_{l m_{\mathrm{II}}}^{(\mathrm{I})} F_{l}^{m_{\mathrm{II}}}-\sum_{m_{\mathrm{I}}}{ }^{j} P_{l m_{\mathrm{I}}}^{(\mathrm{II})} F_{l}^{m_{\mathrm{I}}}=-{ }^{j} \chi_{B}^{\mathrm{in}}, \\
& \sum_{m_{\mathrm{II}}} \sum_{l}{ }_{l}^{j} Q_{l m_{\mathrm{II}}}^{(\mathrm{I})} F_{l}^{m_{\mathrm{II}}}-\sum_{m_{\mathrm{I}}}{ }^{j} Q_{l m_{\mathrm{I}}}^{(\mathrm{II})} F_{l}^{m_{\mathrm{I}}} \\
& \quad=-\left(\cos \phi^{j} \chi_{A}^{\text {in }}+\sin \phi^{j} \chi_{C}^{\mathrm{in}}\right),
\end{aligned}
$$


where

$$
\begin{aligned}
& { }^{j} P_{l m_{\mathrm{II}}}^{(\mathrm{I})}=i \kappa_{0} H_{l}^{(1)}\left(k_{0}\left|\mathbf{r}_{j}-\mathbf{r}_{m_{\mathrm{II}}}\right|\right) e^{i l \theta_{m_{\mathrm{II}}}}, \\
& { }^{j} P_{l m_{\mathrm{I}}}^{(\mathrm{II})}=i \kappa_{1} H_{l}^{(1)}\left(k_{1}\left|\mathbf{r}_{j}-\mathbf{r}_{m_{\mathrm{I}}}\right|\right) e^{i l \theta_{m_{\mathrm{I}}}}, \\
& { }^{j} Q_{l m_{\mathrm{II}}}^{(\mathrm{I})}=\cos ^{2} \phi H_{l-1}^{(1)}\left(k_{0}\left|\mathbf{r}_{j}-\mathbf{r}_{m_{\mathrm{II}}}\right|\right) e^{i(l-1) \theta_{m_{\mathrm{II}}}}-\sin ^{2} \phi H_{l+1}^{(1)}\left(k_{0}\left|\mathbf{r}_{j}-\mathbf{r}_{m_{\mathrm{II}}}\right|\right) e^{i(l+1) \theta_{m_{\mathrm{II}}}}, \\
& { }^{j} Q_{l m_{\mathrm{I}}}^{(\mathrm{II})}=\cos ^{2} \phi H_{l-1}^{(1)}\left(k_{1}\left|\mathbf{r}_{j}-\mathbf{r}_{m_{\mathrm{I}}}\right|\right) e^{i(l-1) \theta_{m_{\mathrm{I}}}}-\sin ^{2} \phi H_{l+1}^{(1)}\left(k_{1}\left|\mathbf{r}_{j}-\mathbf{r}_{m_{\mathrm{I}}}\right|\right) e^{i(l+1) \theta_{m_{\mathrm{I}}}},
\end{aligned}
$$

and

$$
{ }^{j} \chi_{A, B, C}^{\text {in }}=\chi_{A, B, C}^{\text {in }}\left(\mathbf{r}_{j}\right) .
$$

We discretize the boundary into $J$ points. For each point, we truncate the angular momentum as $l \in[-L, L]$. Let $N_{L}=2 L+1$ be the number of eigenvectors associated with different values of the angular momentum and let $N=N_{L} \times\left(M_{\mathrm{I}}+M_{\mathrm{II}}\right)=N_{\mathrm{I}}+N_{\mathrm{II}}$ The boundary conditions lead to the following matrix equation,

$$
\mathbb{M}_{2 J \times N} \cdot \mathbf{F}_{N \times 1}=-\mathbf{G}_{2 J \times 1},
$$

where $\mathbb{M}$ can be expanded in terms of the matrices $\mathbb{P}$ and $\mathbb{Q}$, which are typically not square matrices:

$$
\mathbb{M}_{2 J \times N}=\left(\begin{array}{l|l}
\mathbb{P}^{(\mathrm{I})} & -\mathbb{P}^{(\mathrm{II})} \\
\hline \mathbb{Q}^{(\mathrm{I})} & -\mathbb{Q}^{(\mathrm{II})}
\end{array}\right)_{2 J \times N} .
$$

The two vectors in Eq. (E8) are given by

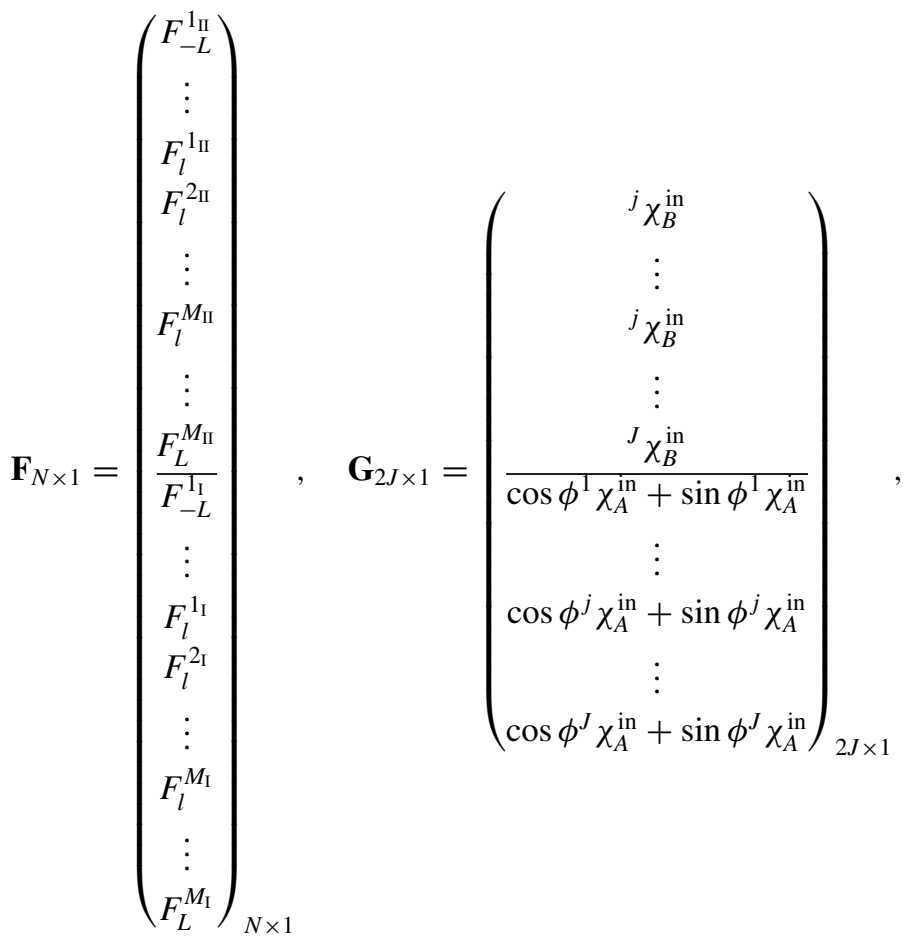

and the matrices $\mathbb{P}$ and $\mathbb{Q}$ are given by

$$
\mathbb{P}^{(\lambda)}=\left(\begin{array}{ccccccccc}
{ }^{1} P_{-L 1_{\bar{\lambda}}}^{(\lambda)} & \ldots & { }^{1} P_{-L M_{\bar{\lambda}}}^{(\lambda)} & \ldots & { }^{1} P_{l 1_{\bar{\lambda}}}^{(\lambda)} & \ldots & { }^{1} P_{l M_{\bar{\lambda}}}^{(\lambda)} & \ldots & { }^{1} P_{L M_{\bar{\lambda}}}^{(\lambda)} \\
{ }^{2} P_{-L 1_{\bar{\lambda}}}^{(\lambda)} & \ldots & { }^{2} P_{-L M_{\bar{\lambda}}}^{(\lambda)} & \ldots & { }^{2} P_{l 1_{\bar{\lambda}}}^{(\lambda)} & \ldots & { }^{2} P_{l M_{\bar{\lambda}}}^{(\lambda)} & \ldots & { }^{2} P_{L M_{\bar{\lambda}}}^{(\lambda)} \\
\vdots & \ldots & \vdots & \ldots & \vdots & \ldots & \vdots & \ldots & \vdots \\
{ }^{j} P_{-L 1_{\bar{\lambda}}}^{(\lambda)} & \ldots & { }^{j} P_{-L M_{\bar{\lambda}}}^{(\lambda)} & \ldots & { }^{j} P_{l 1_{\bar{\lambda}}}^{(\lambda)} & \ldots & { }^{j} P_{l M_{\bar{\lambda}}}^{(\lambda)} & \ldots & { }^{j} P_{L M_{\bar{\lambda}}}^{(\lambda)} \\
\vdots & \ldots & \vdots & \ldots & \vdots & \ldots & \vdots & \ldots & \vdots \\
{ }^{J} P_{-L 1_{\bar{\lambda}}}^{(\lambda)} & \ldots & { }^{J} P_{-L M_{\bar{\lambda}}}^{(\lambda)} & \ldots & { }^{J} P_{l 1_{\bar{\lambda}}}^{(\lambda)} & \ldots & { }^{J} P_{l M_{\bar{\lambda}}}^{(\lambda)} & \ldots & { }^{J} P_{L M_{\bar{\lambda}}}^{(\lambda)}
\end{array}\right)_{J \times N_{\bar{\lambda}}}
$$


and

$$
\mathbb{Q}^{(\lambda)}=\left(\begin{array}{ccccccccc}
{ }^{1} Q_{-L 1_{\bar{\lambda}}}^{(\lambda)} & \ldots & { }^{1} Q_{-L M_{\bar{\lambda}}}^{(\lambda)} & \ldots & { }^{1} Q_{l 1_{\bar{\lambda}}}^{(\lambda)} & \ldots & { }^{1} Q_{l M_{\bar{\lambda}}}^{(\lambda)} & \ldots & { }^{1} Q_{L M_{\bar{\lambda}}}^{(\lambda)} \\
{ }^{2} Q_{-L 1_{\bar{\lambda}}}^{(\lambda)} & \ldots & { }^{2} Q_{-L M_{\bar{\lambda}}}^{(\lambda)} & \ldots & { }^{2} Q_{l 1_{\bar{\lambda}}}^{(\lambda)} & \ldots & { }^{2} Q_{l M_{\bar{\lambda}}}^{(\lambda)} & \ldots & { }^{2} Q_{L M_{\bar{\lambda}}}^{(\lambda)} \\
\vdots & \ldots & \vdots & \ldots & \vdots & \ldots & \vdots & \ldots & \vdots \\
{ }^{j} Q_{-L 1_{\bar{\lambda}}}^{(\lambda)} & \ldots & { }^{j} Q_{-L M_{\bar{\lambda}}}^{(\lambda)} & \ldots & { }^{j} Q_{l 1_{\bar{\lambda}}}^{(\lambda)} & \ldots & { }^{j} Q_{l M_{\bar{\lambda}}}^{(\lambda)} & \ldots & { }^{j} Q_{L M_{\bar{\lambda}}}^{(\lambda)} \\
\vdots & \ldots & \vdots & \ldots & \vdots & \ldots & \vdots & \ldots & \vdots \\
{ }^{J} Q_{-L 1_{\bar{\lambda}}}^{(\lambda)} & \ldots & { }^{J} Q_{-L M_{\bar{\lambda}}}^{(\lambda)} & \ldots & { }^{J} Q_{l 1_{\bar{\lambda}}}^{(\lambda)} & \ldots & { }^{J} Q_{l M_{\bar{\lambda}}}^{(\lambda)} & \ldots & { }^{J} Q_{L M_{\bar{\lambda}}}^{(\lambda)}
\end{array}\right)_{J \times N_{\bar{\lambda}}}
$$

where $\lambda \in\{\mathrm{I}, \mathrm{II}\}$ and $\bar{\lambda}=\{\mathrm{I}, \mathrm{II}\}-\lambda$.

Because of the finite truncation, the total basis is not complete, so Eq. (E9) can be satisfied only approximately. To achieve high accuracy requires $J \gg N$. Equation (E9) can be solved by the pseudoinverse method $[\mathbf{F}=-\operatorname{pinv}(\mathbb{M} * \mathbf{G})$ in MATLAB].

From the expansion coefficients $\mathbf{F}$, we can get the scattering wave function in the whole space. To validate the MMP method, we set $\alpha=1 / 3$ in the circular cavity and calculate the local density of states (LDOS) as a function of the parameter $V R$ both analytically and using the MMP method. The results are shown in Figs. 11(d) and 11(e). There is a good agreement between the results.

The error of the MMP method can be estimated as

$$
\mathrm{SSE}=\frac{\|\mathrm{M} * \mathbf{F}+\mathbf{G}\|}{\|\mathbf{G}\|} .
$$

For the circular and the elliptical cavities, the relative errors are typically smaller than $10^{-5}$. For the stadium cavity, the error is bounded by 0.05 . In the quantum-dot regime where the wave vector inside the cavity is large, a large number of poles are needed and they should be placed as close to the boundary as possible.
[1] K. S. Novoselov, A. K. Geim, S. V. Morozov, D. Jiang, Y. Zhang, S. V. Dubonos, I. V. Grigorieva, and A. A. Firsov, Electric field effect in atomically thin carbon films, Science 306, 666 (2004).

[2] K. S. Novoselov, A. K. Geim, S. V. Morozov, D. Jiang, M. I. Katsnelson, I. V. Grigorieva, S. V. Dubonos, and A. A. Firsov, Two-dimensional gas of massless Dirac fermions in graphene, Nature (London) 438, 197 (2005).

[3] A. H. Castro Neto, F. Guinea, N. M. R. Peres, K. S. Novoselov, and A. K. Geim, The electronic properties of graphene, Rev. Mod. Phys. 81, 109 (2009).

[4] K. S. Novoselov, A. Mishchenko, A. Carvalho, and A. H. C. Neto, 2D materials and van der Waals heterostructures, Science 353, aac9439 (2016).

[5] D. Bercioux, D. F. Urban, H. Grabert, and W. Häusler, Massless Dirac-Weyl fermions in a $T_{3}$ optical lattice, Phys. Rev. A 80, 063603 (2009).

[6] A. Raoux, M. Morigi, J.-N. Fuchs, F. Piéchon, and G. Montambaux, From Dia- to Paramagnetic Orbital Susceptibility of Massless Fermions, Phys. Rev. Lett. 112, 026402 (2014).

[7] E. Illes, J. P. Carbotte, and E. J. Nicol, Hall quantization and optical conductivity evolution with variable Berry phase in the $\alpha-T_{3}$ model, Phys. Rev. B 92, 245410 (2015).

[8] E. Illes and E. J. Nicol, Magnetic properties of the $\alpha-T_{3}$ model: Magneto-optical conductivity and the Hofstadter butterfly, Phys. Rev. B 94, 125435 (2016).

[9] T. Biswas and T. K. Ghosh, Magnetotransport properties of the $\alpha-T_{3}$ model, J. Phys.: Condens. Matter 28, 495302 (2016).

[10] Á. D. Kovács, G. Dávid, B. Dóra, and J. Cserti, Frequencydependent magneto-optical conductivity in the generalized $\alpha-T_{3}$ model, Phys. Rev. B 95, 035414 (2017).
[11] H.-Y. Xu, L. Huang, D. Huang, and Y.-C. Lai, Geometric valley Hall effect and valley filtering through a singular Berry flux, Phys. Rev. B 96, 045412 (2017).

[12] E. Illes and E. J. Nicol, Klein tunneling in the $\alpha-T_{3}$ model, Phys. Rev. B 95, 235432 (2017).

[13] Y. Xu and L.-M. Duan, Unconventional quantum Hall effects in two-dimensional massive spin-1 fermion systems, Phys. Rev. B 96, 155301 (2017).

[14] B. Dey and T. K. Ghosh, Photoinduced valley and electronhole symmetry breaking in $\alpha-T_{3}$ lattice: The role of a variable Berry phase, Phys. Rev. B 98, 075422 (2018).

[15] C.-Z. Wang, C.-D. Han, H.-Y. Xu, and Y.-C. Lai, Chaos-based Berry phase detector, Phys. Rev. B 99, 144302 (2019).

[16] E. V. Gorbar, V. P. Gusynin, and D. O. Oriekhov, Electron states for gapped pseudospin-1 fermions in the field of a charged impurity, Phys. Rev. B 99, 155124 (2019).

[17] M. Rizzi, V. Cataudella, and R. Fazio, Phase diagram of the Bose-Hubbard model with $T_{3}$ symmetry, Phys. Rev. B 73, 144511 (2006).

[18] D. Leykam and S. Flach, Perspective: Photonic flatbands, APL Photon. 3, 070901 (2018).

[19] S. Mukherjee, M. Di Liberto, P. Öhberg, R. R. Thomson, and N. Goldman, Experimental Observation of Aharonov-Bohm Cages in Photonic Lattices, Phys. Rev. Lett. 121, 075502 (2018).

[20] A. Fang, Z. Q. Zhang, S. G. Louie, and C. T. Chan, Klein tunneling and supercollimation of pseudospin-1 electromagnetic waves, Phys. Rev. B 93, 035422 (2016).

[21] F. Wang and Y. Ran, Nearly flat band with Chern number $c=$ 2 on the dice lattice, Phys. Rev. B 84, 241103(R) (2011).

[22] J. Romhányi, K. Penc, and R. Ganesh, Hall effect of triplons in a dimerized quantum magnet, Nat. Commun. 6, 6805 (2015). 
[23] G. Giovannetti, M. Capone, J. van den Brink, and C. Ortix, Kekulé textures, pseudospin-one Dirac cones, and quadratic band crossings in a graphene-hexagonal indium chalcogenide bilayer, Phys. Rev. B 91, 121417(R) (2015).

[24] K. J. Vahala, Optical microcavities, Nature (London) 424, 839 (2003).

[25] W. Han, R. K. Kawakami, M. Gmitra, and J. Fabian, Graphene spintronics, Nat. Nanotechnol. 9, 794 (2014).

[26] A. Rycerz, J. Tworzydło, and C. Beenakker, Valley filter and valley valve in graphene, Nat. Phys. 3, 172 (2007).

[27] C.-D. Han, C.-Z. Wang, H.-Y. Xu, D. Huang, and Y.-C. Lai, Decay of semiclassical massless Dirac fermions from integrable and chaotic cavities, Phys. Rev. B 98, 104308 (2018).

[28] M. I. Katsnelson, K. S. Novoselov, and A. K. Geim, Chiral tunneling and the Klein paradox in graphene, Nat. Phys. 2, 620 (2006).

[29] A. V. Shytov, M. S. Rudner, and L. S. Levitov, Klein Backscattering and Fabry-Pérot Interference in Graphene Heterojunctions, Phys. Rev. Lett. 101, 156804 (2008).

[30] N. Stander, B. Huard, and D. Goldhaber-Gordon, Evidence for Klein Tunneling in Graphene $p-n$ Junctions, Phys. Rev. Lett. 102, 026807 (2009).

[31] A. F. Young and P. Kim, Quantum interference and Klein tunnelling in graphene heterojunctions, Nat. Phys. 5, 222 (2009).

[32] R. Yang, L. Huang, Y.-C. Lai, and C. Grebogi, Abnormal electron paths induced by Klein tunneling in graphene quantum point contacts, Phys. Rev. B 84, 035426 (2011).

[33] H.-Y. Xu and Y.-C. Lai, Revival resonant scattering, perfect caustics, and isotropic transport of pseudospin-1 particles, Phys. Rev. B 94, 165405 (2016).

[34] C. Gutierrez, L. Brown, C.-J. Kim, J. Park, and A. N. Pasupathy, Klein tunnelling and electron trapping in nanometre-scale graphene quantum dots, Nat. Phys. 12, 1069 (2016).

[35] A. Mekis, J. U. Nöckel, G. Chen, A. D. Stone, and R. K. Chang, Ray Chaos and $Q$ Spoiling in Lasing Droplets, Phys. Rev. Lett. 75, 2682 (1995).

[36] J. U. Nöckel and A. D. Stone, Ray and wave chaos in asymmetric resonant optical cavities, Nature (London) 385, 45 (1997).

[37] H. Cao and J. Wiersig, Dielectric microcavities: Model systems for wave chaos and non-Hermitian physics, Rev. Mod. Phys. 87, 61 (2015).

[38] Y. Zhao, J. Wyrick, F. D. Natterer, J. F. Rodriguez-Nieva, C. Lewandowski, K. Watanabe, T. Taniguchi, L. S. Levitov, N. B. Zhitenev, and J. A. Stroscio, Creating and probing electron whispering-gallery modes in graphene, Science $\mathbf{3 4 8}$, 672 (2015)

[39] J. Lee, D. Wong, J. Velasco Jr., J. F. Rodriguez-Nieva, S. Kahn, H.-Z. Tsai, T. Taniguchi, K. Watanabe, A. Zettl, F. Wang et al., Imaging electrostatically confined Dirac fermions in graphene quantum dots, Nat. Phys. 12, 1032 (2016).

[40] F. Ghahari, D. Walkup, C. Gutiérrez, J. F. Rodriguez-Nieva, Y. Zhao, J. Wyrick, F. D. Natterer, W. G. Cullen, K. Watanabe, T. Taniguchi et al., An on/off Berry phase switch in circular graphene resonators, Science 356, 845 (2017).

[41] J. Velasco Jr., L. Ju, D. Wong, S. Kahn, J. Lee, H.-Z. Tsai, C. Germany, S. Wickenburg, J. Lu, T. Taniguchi et al., Nanoscale control of rewriteable doping patterns in pristine graphene/boron nitride heterostructures, Nano Lett. 16, 1620 (2016).

[42] P. E. Allain and J.-N. Fuchs, Klein tunneling in graphene: Optics with massless electrons, Eur. Phys. J. B 83, 301 (2011).

[43] J. Cserti, A. Pályi, and C. Péterfalvi, Caustics Due to a Negative Refractive Index in Circular Graphene $p$ - $n$ Junctions, Phys. Rev. Lett. 99, 246801 (2007).

[44] V. V. Cheianov, V. Fal'ko, and B. Altshuler, The focusing of electron flow and a Veselago lens in graphene $p$ - $n$ junctions, Science 315, 1252 (2007).

[45] X. Du, I. Skachko, A. Barker, and E. Y. Andrei, Approaching ballistic transport in suspended graphene, Nat. Nanotechnol. 3, 491 (2008).

[46] C. W. J. Beenakker, R. A. Sepkhanov, A. R. Akhmerov, and J. Tworzydło, Quantum Goos-Hänchen Effect in Graphene, Phys. Rev. Lett. 102, 146804 (2009).

[47] A. G. Moghaddam and M. Zareyan, Graphene-Based Electronic Spin Lenses, Phys. Rev. Lett. 105, 146803 (2010).

[48] N. Gu, M. Rudner, and L. Levitov, Chirality-Assisted Electronic Cloaking of Confined States in Bilayer Graphene, Phys. Rev. Lett. 107, 156603 (2011).

[49] J. Williams, T. Low, M. Lundstrom, and C. Marcus, Gatecontrolled guiding of electrons in graphene, Nat. Nanotechnol. 6, 222 (2011).

[50] P. Rickhaus, R. Maurand, M.-H. Liu, M. Weiss, K. Richter, and C. Schönenberger, Ballistic interferences in suspended graphene, Nat. Commun. 4, 2342 (2013).

[51] B. Liao, M. Zebarjadi, K. Esfarjani, and G. Chen, Isotropic and energy-selective electron cloaks on graphene, Phys. Rev. B 88, 155432 (2013).

[52] R. L. Heinisch, F. X. Bronold, and H. Fehske, Mie scattering analog in graphene: Lensing, particle confinement, and depletion of Klein tunneling, Phys. Rev. B 87, 155409 (2013).

[53] M. M. Asmar and S. E. Ulloa, Rashba spin-orbit interaction and birefringent electron optics in graphene, Phys. Rev. B 87, 075420 (2013).

[54] J.-S. Wu and M. M. Fogler, Scattering of two-dimensional massless Dirac electrons by a circular potential barrier, Phys. Rev. B 90, 235402 (2014).

[55] P. Rickhaus, M.-H. Liu, P. Makk, R. Maurand, S. Hess, S. Zihlmann, M. Weiss, K. Richter, and C. Schönenberger, Guiding of electrons in a few-mode ballistic graphene channel, Nano Lett. 15, 5819 (2015).

[56] G.-H. Lee, G.-H. Park, and H.-J. Lee, Observation of negative refraction of Dirac fermions in graphene, Nat. Phys. 11, 925 (2015).

[57] P. Rickhaus, P. Makk, M.-H. Liu, K. Richter, and C. Schönenberger, Gate tuneable beamsplitter in ballistic graphene, Appl. Phys. Lett. 107, 251901 (2015).

[58] J. D. Walls and D. Hadad, The Talbot effect for twodimensional massless Dirac fermions, Sci. Rep. 6, 26698 (2016).

[59] J. M. Caridad, S. Connaughton, C. Ott, H. B. Weber, and V. Krstić, An electrical analogy to Mie scattering, Nat. Commun. 7, 12894 (2016).

[60] S. Chen, Z. Han, M. M. Elahi, K. M. Habib, L. Wang, B. Wen, Y. Gao, T. Taniguchi, K. Watanabe, J. Hone et al., Electron optics with $p n$ junctions in ballistic graphene, Science 353, 1522 (2016). 
[61] M. Settnes, S. R. Power, M. Brandbyge, and A.-P. Jauho, Graphene Nanobubbles as Valley Filters and Beam Splitters, Phys. Rev. Lett. 117, 276801 (2016).

[62] M.-H. Liu, C. Gorini, and K. Richter, Creating and Steering Highly Directional Electron Beams in Graphene, Phys. Rev. Lett. 118, 066801 (2017).

[63] A. W. Barnard, A. Hughes, A. L. Sharpe, K. Watanabe, T. Taniguchi, and D. Goldhaber-Gordon, Absorptive pinhole collimators for ballistic Dirac fermions in graphene, Nat. Commun. 8, 15418 (2017).

[64] Y. Jiang, J. Mao, D. Moldovan, M. R. Masir, G. Li, K. Watanabe, T. Taniguchi, F. M. Peeters, and E. Y. Andrei, Tuning a circular $p$ - $n$ junction in graphene from quantum confinement to optical guiding, Nat. Nanotechnol. 12, 1045 (2017).

[65] S.-H. Zhang, J.-J. Zhu, W. Yang, and K. Chang, Focusing RKKY interaction by graphene $p-n$ junction, 2D Mater. 4, 035005 (2017).

[66] P. Bøggild, J. M. Caridad, C. Stampfer, G. Calogero, N. R. Papior, and M. Brandbyge, A two-dimensional Dirac fermion microscope, Nat. Commun. 8, 15783 (2017).

[67] H.-Y. Xu, G.-L. Wang, L. Huang, and Y.-C. Lai, Chaos in Dirac Electron Optics: Emergence of a Relativistic Quantum Chimera, Phys. Rev. Lett. 120, 124101 (2018).

[68] D. S. Novikov, Elastic scattering theory and transport in graphene, Phys. Rev. B 76, 245435 (2007).

[69] C. A. Downing, A. R. Pearce, R. J. Churchill, and M. E. Portnoi, Optimal traps in graphene, Phys. Rev. B 92, 165401 (2015).

[70] H. Xu and Y.-C. Lai, Superscattering of a pseudospin-1 wave in a photonic lattice, Phys. Rev. A 95, 012119 (2017).

[71] E. P. Wigner, Lower limit for the energy derivative of the scattering phase shift, Phys. Rev. 98, 145 (1955).

[72] F. T. Smith, Lifetime matrix in collision theory, Phys. Rev. 118, 349 (1960).

[73] S. Rotter and S. Gigan, Light fields in complex media: Mesoscopic scattering meets wave control, Rev. Mod. Phys. 89, 015005 (2017).

[74] T. Ando, T. Nakanishi, and R. Saito, Berry's phase and absence of back scattering in carbon nanotubes, J. Phys. Soc. Jpn. 67, 2857 (1998).

[75] D. Basko, Resonant low-energy electron scattering on shortrange impurities in graphene, Phys. Rev. B 78, 115432 (2008).

[76] M. Titov, P. M. Ostrovsky, I. V. Gornyi, A. Schuessler, and A. D. Mirlin, Charge Transport in Graphene with Resonant Scatterers, Phys. Rev. Lett. 104, 076802 (2010).

[77] M. Hentschel and K. Richter, Quantum chaos in optical systems: The annular billiard, Phys. Rev. E 66, 056207 (2002).

[78] A. Bäcker, R. Ketzmerick, S. Löck, J. Wiersig, and M. Hentschel, Quality factors and dynamical tunneling in annular microcavities, Phys. Rev. A 79, 063804 (2009).

[79] A. Yamilov and H. Cao, Density of resonant states and a manifestation of photonic band structure in small clusters of spherical particles, Phys. Rev. B 68, 085111 (2003).

[80] M. Davy, Z. Shi, J. Wang, X. Cheng, and A. Z. Genack, Transmission Eigenchannels and the Densities of States of Random Media, Phys. Rev. Lett. 114, 033901 (2015).

[81] H.-J. Stöckmann, Quantum Chaos: An Introduction (Cambridge University Press, New York, 1999).
[82] Y.-C. Lai, H.-Y. Xu, L. Huang, and C. Grebogi, Relativistic quantum chaos: an emergent interdisciplinary field, Chaos 28, 052101 (2018).

[83] L. Huang, H.-Y. Xu, C. Grebogi, and Y.-C. Lai, Relativistic quantum chaos, Phys. Rep. 753, 1 (2018).

[84] R. Blümel and U. Smilansky, Classical Irregular Scattering and its Quantum-Mechanical Implications, Phys. Rev. Lett. 60, 477 (1988).

[85] R. Blümel and U. Smilansky, A simple model for chaotic scattering: II. Quantum mechanical theory, Phys. D (Amsterdam, Neth.) 36, 111 (1989).

[86] Y.-C. Lai, R. Blümel, E. Ott, and C. Grebogi, Quantum Manifestations of Chaotic Scattering, Phys. Rev. Lett. 68, 3491 (1992).

[87] R. Ketzmerick, Fractal conductance fluctuations in generic chaotic cavities, Phys. Rev. B 54, 10841 (1996).

[88] A. P. S. de Moura, Y.-C. Lai, R. Akis, J. P. Bird, and D. K. Ferry, Tunneling and Nonhyperbolicity in Quantum Dots, Phys. Rev. Lett. 88, 236804 (2002).

[89] R. Yang, L. Huang, Y.-C. Lai, and C. Grebogi, Quantum chaotic scattering in graphene systems, Europhys. Lett. 94, 40004 (2011).

[90] J. H. Bardarson, M. Titov, and P. W. Brouwer, Electrostatic Confinement of Electrons in an Integrable Graphene Quantum Dot, Phys. Rev. Lett. 102, 226803 (2009).

[91] M. Schneider and P. W. Brouwer, Resonant scattering in graphene with a gate-defined chaotic quantum dot, Phys. Rev. B 84, 115440 (2011).

[92] J. Heinl, M. Schneider, and P. W. Brouwer, Interplay of Aharonov-Bohm and Berry phases in gate-defined graphene quantum dots, Phys. Rev. B 87, 245426 (2013).

[93] M. Schneider and P. W. Brouwer, Density of states as a probe of electrostatic confinement in graphene, Phys. Rev. B 89, 205437 (2014).

[94] H.-Y. Xu and Y.-C. Lai, Pseudospin-1 wave scattering that defies chaos $Q$ spoiling and Klein tunneling, Phys. Rev. B 99, 235403 (2019).

[95] Y. Leviatan and A. Boag, Analysis of electromagnetic scattering from dielectric cylinders using a multifilament current model, IEEE Trans. Antennas Propag. 35, 1119 (1987).

[96] M. G. Imhof, Multiple multipole expansions for elastic scattering, J. Acoust. Soc. Am. 100, 2969 (1996).

[97] D. I. Kaklamani and H. T. Anastassiu, Aspects of the method of auxiliary sources (MAS) in computational electromagnetics, IEEE Anten. Propag. Maga. 44, 48 (2002).

[98] E. Moreno, D. Erni, C. Hafner, and R. Vahldieck, Multiple multipole method with automatic multipole setting applied to the simulation of surface plasmons in metallic nanostructures, J. Opt. Soc. Am. A 19, 101 (2002).

[99] G. Tayeb and S. Enoch, Combined fictitious-sourcesscattering-matrix method, J. Opt. Soc. Am. A 21, 1417 (2004).

[100] E. J. Heller, Bound-State Eigenfunctions of Classically Chaotic Hamiltonian Systems: Scars of Periodic Orbits, Phys. Rev. Lett. 53, 1515 (1984).

[101] F. Haake, Quantum Signatures of Chaos, 3rd ed., Springer Series in Synergetics (Springer-Verlag, Berlin, 2010).

[102] L. Huang, Y.-C. Lai, D. K. Ferry, S. M. Goodnick, and R. Akis, Relativistic Quantum Scars, Phys. Rev. Lett. 103, 054101 (2009). 
[103] M. V. Berry and R. Mondragon, Neutrino billiards: Timereversal symmetry-breaking without magnetic fields, Proc. R. Soc. London, Ser. A 412, 53 (1987).

[104] H. Xu, L. Huang, Y.-C. Lai, and C. Grebogi, Chiral Scars in Chaotic Dirac Fermion Systems, Phys. Rev. Lett. 110, 064102 (2013).

[105] C.-Z. Wang, L. Huang, and K. Chang, Scars in Dirac fermion systems: The influence of an Aharonov-Bohm flux, New J. Phys. 19, 013018 (2017).

[106] M.-Y. Song, Z.-Y. Li, H.-Y. Xu, L. Huang, and Y.-C. Lai, Quantization of massive Dirac billiards and unification of nonrelativistic and relativistic chiral quantum scars, Phys. Rev. Research 1, 033008 (2019).

[107] J. Wiersig and M. Hentschel, Combining Directional Light Output and Ultralow Loss in Deformed Microdisks, Phys. Rev. Lett. 100, 033901 (2008).
[108] S. Shinohara, T. Harayama, T. Fukushima, M. Hentschel, S. Sunada, and E. E. Narimanov, Chaos-assisted emission from asymmetric resonant cavity microlasers, Phys. Rev. A 83, 053837 (2011).

[109] K. Chang and W.-K. Lou, Helical Quantum States in HgTe Quantum Dots with Inverted Band Structures, Phys. Rev. Lett. 106, 206802 (2011).

[110] A. Tadjine and C. Delerue, Topological protection of electronic states against disorder probed by their magnetic moment, Phys. Rev. B 95, 235426 (2017).

[111] B. Szafran, A. Mreńca-Kolasińska, B. Rzeszotarski, and D. Żebrowski, Electrical control of a confined electron spin in a silicene quantum dot, Phys. Rev. B 97, 165303 (2018).

[112] F. W. Olver, D. W. Lozier, R. F. Boisvert, and C. W. Clark, NIST Handbook of Mathematical Functions (Cambridge University Press, New York, 2010). 